\title{
The Fan Region at 1.5 GHz - I. Polarized synchrotron emission extending beyond the Perseus Arm
}

\author{
A. S. Hill, ${ }^{1,2 \star}$ T. L. Landecker, ${ }^{3}$ E. Carretti,${ }^{1} \dagger$ K. Douglas, ${ }^{4}$ X. H. Sun,${ }^{5}$ \\ B. M. Gaensler, ${ }^{5,6}$ S. A. Mao, ${ }^{7}$ N. M. McClure-Griffiths, ${ }^{1,8}$ W. Reich, ${ }^{7}$ \\ M. Wolleben, ${ }^{9}$ J. M. Dickey, ${ }^{10}$ A. D. Gray, ${ }^{3}$ M. Haverkorn, ${ }^{11}$ J. P. Leahy ${ }^{12}$ \\ and D. H. F. M. Schnitzeler ${ }^{7}$ \\ ${ }^{1}$ CSIRO Astronomy \& Space Science, PO Box 76, Epping, NSW 1710, Australia \\ ${ }^{2}$ Department of Astronomy, Haverford College, Haverford, PA 19041, USA \\ ${ }^{3}$ National Research Council Canada, Herzberg Program in Astronomy and Astrophysics, Dominion Radio Astrophysical Observatory, PO Box 248, Penticton, \\ British Columbia V2A 6J9, Canada \\ ${ }^{4}$ Physics and Astronomy Department, Okanagan College, 1000 KLO Road, Kelowna, British Columbia VIY 4X8, Canada \\ ${ }^{5}$ Sydney Institute for Astronomy, School of Physics, University of Sydney, NSW 2006, Australia \\ ${ }^{6}$ Dunlap Institute for Astronomy and Astrophysics, University of Toronto, 50 St. George Street, Toronto, Ontario M5S 3H4, Canada \\ ${ }^{7}$ Max-Planck-Institut für Radioastronomie, Auf dem Hügel 69, D-53121, Bonn, Germany \\ ${ }^{8}$ Research School of Astronomy and Astrophysics, Australian National University, Canberra, ACT 2611, Australia \\ ${ }^{9}$ Skaha Remote Sensing, 3165 Juniper Drive, Naramata, BC VOH 1NO, Canada \\ ${ }^{10}$ University of Tasmania, School of Mathematics and Physics, Hobart, TAS 7001, Australia \\ ${ }^{11}$ Department of Astrophysics/IMAPP, Radboud University Nijmegen, PO Box 9010, NL-6500 GL, Nijmegen, the Netherlands \\ ${ }^{12}$ Jodrell Bank Centre for Astrophysics, Alan Turing Building, School of Physics and Astronomy, University of Manchester, Oxford Road, Manchester \\ M13 9PL, UK
}

Accepted 2017 February 13. Received 2017 February 13; in original form 2016 September 1

\begin{abstract}
A BST RA CT
The Fan Region is one of the dominant features in the polarized radio sky, long thought to be a local (distance $\lesssim 500 \mathrm{pc}$ ) synchrotron feature. We present $1.3-1.8 \mathrm{GHz}$ polarized radio continuum observations of the region from the Global Magneto-Ionic Medium Survey and compare them to maps of $\mathrm{H} \alpha$ and polarized radio continuum intensity from 0.408 to $353 \mathrm{GHz}$. The high-frequency $(>1 \mathrm{GHz})$ and low-frequency $(\lesssim 600 \mathrm{MHz})$ emissions have different morphologies, suggesting a different physical origin. Portions of the $1.5 \mathrm{GHz}$ Fan Region emission are depolarized by $\approx 30$ per cent by ionized gas structures in the Perseus Arm, indicating that this fraction of the emission originates $\gtrsim 2 \mathrm{kpc}$ away. We argue for the same conclusion based on the high polarization fraction at $1.5 \mathrm{GHz}(\approx 40$ per cent). The Fan Region is offset with respect to the Galactic plane, covering $-5^{\circ} \lesssim b \lesssim+10^{\circ}$; we attribute this offset to the warp in the outer Galaxy. We discuss origins of the polarized emission, including the spiral Galactic magnetic field. This idea is a plausible contributing factor although no model to date readily reproduces all of the observations. We conclude that models of the Galactic magnetic field should account for the $\gtrsim 1 \mathrm{GHz}$ emission from the Fan Region as a Galactic scale, not purely local, feature.
\end{abstract}

Key words: polarization-ISM: magnetic fields-ISM: structure-Galaxy: structure-radio continuum: ISM.

\section{INTRODUCTION}

Linearly polarized radio continuum emission arises from the interaction of ultrarelativistic electrons with a magnetic field. The

\footnotetext{
*E-mail: ashill@ haverford.edu

† Present address: INAF/Osservatorio Astronomico di Cagliari, Via della

Scienza 5, I-09047 Selargius, Italy.
}

first detections of polarized emission from the Galaxy (Westerhout et al. 1962; Wielebinski, Shakeshaft \& Pauliny-Toth 1962) already recognized the two large features that dominate the northern polarized sky, the North Polar Spur and the Fan Region. The Fan Region extends over an $\sim 60^{\circ} \times 30^{\circ}$ region, centred at Galactic longitude $\ell \approx 130^{\circ}$ slightly above the Galactic plane at Galactic latitude $b \approx+5^{\circ}$. The Fan Region is remarkable for the intensity of its polarized radiation and the regularity of its polarization angle. In total intensity images, it does not stand out from its 
surroundings. It is identified by (and named for) electric field vectors that appear to fan out from the Galactic plane near $\ell=130^{\circ}$ at low frequencies $v \lesssim 600 \mathrm{MHz}$ (Bingham \& Shakeshaft 1967; Brouw \& Spoelstra 1976). Polarized emission in this region of the sky is evident from $\approx 100 \mathrm{MHz}$ (Iacobelli, Haverkorn \& Katgert 2013a) to $353 \mathrm{GHz}$ (Planck Collaboration XIX 2015). All of this emission is generally referred to as the Fan Region.

The origin of the Fan Region is unknown, but most authors have considered it a local $(d \lesssim 500$ pc) feature (Wilkinson \& Smith 1974; Spoelstra 1984). Verschuur (1968) identified a depolarized ring feature at $408 \mathrm{MHz}$ at $\left(137^{\circ},+7^{\circ}\right)^{1}$, which they associated with a star $140-200 \mathrm{pc}$ from the Sun to establish a lower limit to the distance; Iacobelli et al. (2013a) placed this ring $\approx 200$ pc away based on $150-350 \mathrm{MHz}$ observations. Wilkinson \& Smith (1974) found no depolarization at $v \leq 610 \mathrm{MHz}$ due to the $\mathrm{H}$ II region Sh2202, establishing an upper limit. The modern distance to Sh2-202 is $0.97 \pm 0.08 \mathrm{kpc}$ (Foster \& Brunt 2015). These arguments for a local origin of the Fan Region are based primarily on low-frequency observations. In contrast, Bingham \& Shakeshaft (1967) argued that the high polarization fraction at $1407 \mathrm{MHz}$ can only be produced by Galactic structure. Wolleben (2005) found depolarization by numerous $\mathrm{H}$ II regions and argued that the $1.4 \mathrm{GHz}$ emission occurs over a range of distances from $\approx 500 \mathrm{pc}$ to a few $\mathrm{kpc}$, a range that includes both local gas and the Perseus spiral arm. Because the intrinsic polarization angle of synchrotron radiation is related to the orientation on the sky of the magnetic field in the emitting region, if the Fan Region emission originates over this long path length, it must indicate a uniform Galactic magnetic field on kpc scales in this direction (Wolleben et al. 2006).

In this paper, we present $1.5 \mathrm{GHz}$ polarized continuum observations of the Fan Region. Our focus here is on morphological comparisons between continuum observations from 0.4 to $353 \mathrm{GHz}$ and with spectroscopically resolved $\mathrm{H} \alpha$ observations. We present our data in Section 2, briefly reviewing relevant depolarization mechanisms in Section 2.3. We discuss the kinematic features seen in $\mathrm{H} \alpha$ and $\mathrm{H}_{\mathrm{I}}$ observations in the direction of the Fan Region as they relate to Galactic structure in Section 3. We describe the Fan Region at all wavelengths and compare the morphology to observations of interstellar medium (ISM) structures with known distances in Section 4. In Section 5.1, we discuss the implications of the high observed fractional polarization in the Fan Region. In Sections 5.2 and 5.3, we construct a simple model of the synchrotron emission due to Galactic spiral structure, incorporating the effects of geometrical and depth depolarization, and compare the results to the observed synchrotron intensity as a function of longitude. We summarize the paper and draw conclusions in Section 6. In Paper II (Hill et al., in preparation), we will model depolarization due to Faraday effects in the Fan Region.

\section{OBSERVATIONS}

\subsection{Global Magneto-Ionic Medium Survey (GMIMS)}

We use radio polarization data from the GMIMS high-band north (GMIMS-HBN). In GMIMS (Wolleben et al. 2009), we are using telescopes around the world to map polarized emission from the entire sky, north and south, spanning 300 to $1800 \mathrm{MHz}$. The survey

\footnotetext{
${ }^{1}$ We denote positions in Galactic coordinates as $(\ell, b)$.
}

is designed to measure the polarized intensity, $L(\phi)$, as a function of Faraday depth

$\phi(s)=K \int_{s}^{\text {observer }} n_{\mathrm{e}}\left(s^{\prime}\right) \boldsymbol{B}\left(s^{\prime}\right) \cdot \mathrm{d} \boldsymbol{s}^{\prime}$.

Here $K \equiv e^{3} /\left(2 \pi m_{\mathrm{e}}^{2} c^{4}\right)=0.81\left(\mathrm{~cm}^{-3} \mu \mathrm{G} \mathrm{pc}\right)^{-1} \mathrm{rad} \mathrm{m}^{-2}, n_{\mathrm{e}}$ is the electron density in the intervening ISM and $\boldsymbol{B}$ is the magnetic field. We acquire data in thousands of frequency channels to allow us to use rotation measure (RM) synthesis (Brentjens \& de Bruyn 2005).

The GMIMS-HBN data were acquired with the $26 \mathrm{~m}$ John A. Galt Telescope at the Dominion Radio Astrophysical Observatory (DRAO) with continuous frequency coverage from 1280 to $1750 \mathrm{MHz}$. Data were acquired in 2048 individual channels of width $236.8 \mathrm{kHz}$. Wolleben et al. (2010b) describe the receiver and the data-acquisition process for GMIMS-HBN, and Wolleben et al. (2010a) and Sun et al. (2015) give examples of use of GMIMSHBN data. The full survey will be presented and publicly released elsewhere.

The observations were made by moving the telescope slowly up and down the meridian as the sky moved by; each such telescope track is referred to as a 'scan'. Earth rotation during a scan caused each scan to follow a diagonal track across the equatorial coordinate grid. Successive up and down scans were made until the sky was fully sampled between declinations $-30^{\circ}$ and $+87^{\circ}$. After calibration, the many scan crossings were reconciled using the 'basketweaving' technique (Wolleben et al. 2010a), which we used to iteratively deduce the best-fitting zero level for each scan. This process strips the sky minimum, which makes the zero-point for Stokes I measurements inconsistent (Wolleben et al. 2010a), so we do not use total intensity data from GMIMS-HBN. Observations were made between sunset and sunrise to avoid contamination through sidelobes by radio emission from the Sun. The angular resolution varies from 40 arcmin to 30 arcmin across this frequency range; we have smoothed the data to a common resolution of 40 arcmin and reprojected to a plate carrée projection (Calabretta \& Greisen 2002).

Daily calibration observations were made of the bright smalldiameter sources Cas A, Cyg A, Tau A and Vir A. Using flux densities and spectral indices of these sources from Baars et al. (1977), we converted the scan data to units of Janskys. The conversion factor from Janksys to Kelvins of main beam antenna temperature (equivalent to the gain of the telescope) was established from careful measurement of the antenna temperature produced by Cyg A on an absolute temperature scale established with resistive terminations at liquid nitrogen temperature and at $\approx 100^{\circ} \mathrm{C}$. Finally, data were converted to main-beam brightness temperatures by dividing by the beam efficiency of the telescope. We consider the temperature scale to be correct within $\sim 3$ per cent. Du et al. (2016) present details of the determination of telescope gain.

The Stokes $Q$ and $U$ spectra are smooth and there is no evidence of bandwidth depolarization in individual channels. Sun et al. (2015) made tests of data quality from the GMIMS data in the vicinity of the North Polar Spur, another region of bright polarized emission. They concluded that the data set is of high quality in regions of bright polarized emission.

We apply RM synthesis to our polarization data cubes. In RM synthesis, we construct the Faraday dispersion function, which takes the form of a Fourier transform of the observed complex polarization vector, $\mathcal{P}=Q+i U$. The Faraday dispersion function is integrated over the interference-free portions of the observed band. At a given Faraday depth, RM synthesis accounts for rotation in the polarization angle over the band. Due to Faraday rotation, there is no single 
Table 1. Data sets used. Bandwidths and channel widths are listed in frequency units for continuum surveys and velocity units for spectral line surveys. The sampling column lists the approximate typical beam spacing on the sky in the mid-plane for surveys that are not Nyquist sampled. References: Dwingeloo: Brouw \& Spoelstra (1976); Carretti et al. (2005). Stockert/Villa Elisa: Reich (1982); Reich \& Reich (1986); Reich, Testori \& Reich (2001). CGPS: Taylor et al. (2003); Landecker et al. (2010). LAB: Kalberla et al. (2005). GMIMS-HBN: This work. Urumqi: Gao et al. (2010). WMAP: Bennett et al. (2013). Planck: Tauber et al. (2010); Planck Collaboration I (2016). WHAM-SS: Haffner et al. (2003, 2010).

\begin{tabular}{|c|c|c|c|c|c|c|c|}
\hline Survey & $\begin{array}{l}\text { Stokes/ } \\
\text { Line }\end{array}$ & $\begin{array}{l}\text { Frequency/ } \\
\text { Wavelength }\end{array}$ & Bandwidth & $\begin{array}{l}\text { Channel } \\
\text { width }\end{array}$ & $\begin{array}{c}\text { Beam } \\
\text { FWHM }\end{array}$ & Sampling & $\begin{array}{l}\text { Coverage } \\
\text { near Fan }\end{array}$ \\
\hline Dwingeloo & $Q, U$ & $408 \mathrm{MHz}$ & $2 \mathrm{MHz}$ & $2 \mathrm{MHz}$ & 2.3 & 2.3 (irregular) & Northern sky \\
\hline Dwingeloo & $Q, U$ & $610 \mathrm{MHz}$ & $4 \mathrm{MHz}$ & $4 \mathrm{MHz}$ & 1.5 & 2.3 (irregular) & Northern sky \\
\hline Dwingeloo & $Q, U$ & $820 \mathrm{MHz}$ & $4 \mathrm{MHz}$ & $4 \mathrm{MHz}$ & 1.0 & 2.3 (irregular) & Northern sky \\
\hline Dwingeloo & $Q, U$ & $1411 \mathrm{MHz}$ & $7 \mathrm{MHz}$ & $7 \mathrm{MHz}$ & 0.6 & 2.3 (irregular) & Northern sky \\
\hline Stockert/Villa Elisa & $I$ & $1420 \mathrm{MHz}$ & $18 \mathrm{MHz}$ & $18 \mathrm{MHz}$ & 0.6 & Nyquist & All sky \\
\hline LAB & $\mathrm{HI}_{\mathrm{I}}$ & $1421 \mathrm{MHz}$ & $\pm 400 \mathrm{~km} \mathrm{~s}^{-1}$ & $1 \mathrm{~km} \mathrm{~s}^{-1}$ & 0.6 & 0.5 & All sky \\
\hline GMIMS-HBN & $Q, U$ & $1500 \mathrm{MHz}$ & $470 \mathrm{MHz}$ & $0.2 \mathrm{MHz}$ & 0.6 & Nyquist & $-30^{\circ}<\delta<+87^{\circ}$ \\
\hline Urumqi & $\widetilde{I}, Q, U$ & $4800 \mathrm{MHz}$ & $600 \mathrm{MHz}$ & $600 \mathrm{MHz}$ & 0.16 & Nyquist & W4 superbubble \\
\hline WMAP & $I, Q, U$ & $22.8 \mathrm{GHz}$ & $5.5 \mathrm{GHz}$ & $5.5 \mathrm{GHz}$ & 0.82 & Nyquist & All sky \\
\hline Planck & $I, \widetilde{Q}, U$ & $353 \mathrm{GHz}$ & $116 \mathrm{GHz}$ & $116 \mathrm{GHz}$ & 0.08 & Nyquist & All sky \\
\hline WHAM-SS & $\mathrm{H} \alpha$ & $656.3 \mathrm{~nm}$ & $\pm 80 \mathrm{~km} \mathrm{~s}^{-1}$ & $12 \mathrm{~km} \mathrm{~s}^{-1}$ & 1.0 & 1.0 & All sky \\
\hline
\end{tabular}

polarization angle that describes the data at all frequencies in the band. Without RM synthesis, integrating over many channels would lead to significant bandwidth depolarization (e.g. Brentjens \& de Bruyn 2005; Heald 2009; Schnitzeler, Katgert \& de Bruyn 2009).

RM synthesis also allows us to separate the emission as a function of $\phi$. For each pixel on the sky, we construct the polarized intensity $L_{1.5}(\phi)$ sampled every $5 \mathrm{rad} \mathrm{m}^{-2}$. Our observing frequencies and spectral resolution leave us sensitive to emission with $|\phi|<2 \times 10^{5} \mathrm{rad} \mathrm{m}^{-2}$; the resolution is $\delta \phi=149 \mathrm{rad} \mathrm{m}^{-2}$ (calculated following Schnitzeler et al. 2009). The data were recorded and Faraday depth spectra calculated in equatorial coordinates; we subsequently reprojected to Galactic coordinates. From each Faraday depth spectrum, we calculated the peak polarized intensity at each pixel using a three-point quadratic fit with the miriad task moment. The polarized intensity images we present in this paper are images of this peak polarized intensity. The Faraday depth of the peak is typically at $|\phi|<10 \mathrm{rad} \mathrm{m}^{-2}$, so these images are similar to an image of the $\phi=0 \mathrm{rad} \mathrm{m}^{-2}$ channel. With the large $\delta \phi$, we assume that there is only a single component resolved by the GMIMS-HBN observations.

When all of the polarized signal is at a single Faraday depth, an image produced with RM synthesis has the noise expected from the entire band rather than the noise from individual frequency channels. The noise in each $\phi$ channel is $\sigma_{L} \approx 0.02 \mathrm{~K}$, making the signal-to-noise ratio in the Fan Region $\gtrsim 20$ on a single-pixel basis. This allows us to measure the centroid of Faraday depth components with an uncertainty of $\approx 3 \mathrm{rad} \mathrm{m}^{-2}$ in the Fan Region. The structures in Faraday depth in the Fan Region seen in low-frequency observations are typically $\sim 1-10 \mathrm{rad} \mathrm{m}^{-2}$ in extent (Iacobelli et al. 2013b), so we do not resolve multiple Faraday depth components with the GMIMS-HBN data; a future low-frequency component of the GMIMS survey will enable the separation of these narrow Faraday depth components. With a maximum frequency of $1750 \mathrm{MHz}$, we are not sensitive to individual Faraday depth features that are wider than $\approx 107 \mathrm{rad} \mathrm{m}^{-2}$ (Brentjens \& de Bruyn 2005).

Wolleben et al. (2006) presented an absolutely calibrated $1.4 \mathrm{GHz}$ polarization survey also using John A. Galt Telescope observations. This older survey employed a single channel of bandwidth $12 \mathrm{MHz}$ and a drift scanning strategy. Each drift scan was Nyquist sampled in right ascension but the survey achieved 41.7 per cent of Nyquist sampling in declination. The GMIMS-HBN survey improves upon the Wolleben et al. (2006) survey with a much wider bandwidth and full Nyquist sampling. Moreover, with the basketweaving observing strategy, each point is observed twice, reducing uncertainty relative to the drift scan strategy.

\subsection{Complementary data sets}

In addition to the GMIMS data presented here, we use several published data sets that provide complementary information. To trace synchrotron emission adequately, we have chosen data sets at a range of frequencies because depolarization cannot be understood from one frequency alone. We have also chosen data sets that trace the dust and ionized and neutral gas (with spectral resolution allowing separation of emission due to Galactic rotation) in the diffuse ISM. We have regridded all of the data sets to a plate carrée projection image with 0.5 pixels. We list frequencies, bandwidths, beam sizes, sampling and the coverage as is relevant to the Fan Region of each of these surveys in Table 1 . We refer the reader to the references in Table 1 for details but mention the most important points for our work here.

We use spectroscopic maps of $\mathrm{H} \alpha$ emission from data release 1 of the all-sky Wisconsin H-Alpha Mapper Sky Survey (WHAM$\mathrm{SS})^{2}$ and of $\mathrm{HI}$ emission from the Leiden-Argentine-Bonn (LAB) survey. We use the low-frequency continuum surveys of Brouw \& Spoelstra (1976), compiled and resampled on a regularly gridded map by Carretti et al. (2005). These observations used rotating feed antennas and thus record the polarized intensity and polarization angle directly (Berkhuijsen et al. 1964). Especially at the higher frequencies, these data are severely undersampled (see Table 1). We use $23 \mathrm{GHz}$ data from the nine-year data release of the Wilkinson Microwave Anisotropy Probe (WMAP) experiment. The high frequency makes these data virtually free from Faraday rotation effects, either angle rotation or depolarization. For the Urumqi $4.8 \mathrm{GHz}$ observations, Gao et al. (2010) set the zero level by extrapolating the WMAP $23 \mathrm{GHz}$ data using a spectral index measured from $23 \mathrm{GHz}$ (WMAP) to $1.4 \mathrm{GHz}$ (Wolleben et al. 2006). This assumes that there

\footnotetext{
${ }^{2}$ http://www.astro.wisc.edu/wham/
} 
is little Faraday depolarization across the band (Sun et al. 2007), an assumption supported by consistent spectral indices. However, the Wolleben et al. (2006) data do not show the depolarization effects that are evident in the GMIMS-HBN data presented here (see Section 4.1 below). If there is any Faraday depolarization at $4.8 \mathrm{GHz}$ on scales larger than a few degrees (where the scaled $23 \mathrm{GHz}$ data sets the zero level), the Urumqi $4.8 \mathrm{GHz}$ data would not be sensitive to it.

In the $353 \mathrm{GHz}$ image from the Planck mission, the thermal emission from dust dominates over other contributions, and the polarization of the signal traces the Galactic magnetic field (Planck Collaboration XIX 2015). The Canadian Galactic Plane Survey (CGPS) provides high angular resolution polarized radio continuum data at $1420 \mathrm{MHz}$ for a small portion of the Fan Region using the DRAO Synthesis Telescope. The Wolleben et al. (2006) data provide information on the largest angular scales for the CGPS data; Effelsberg $100 \mathrm{~m}$ telescope data provide information on intermediate scales.

\subsection{Synchrotron emission and depolarization}

The polarized radio continuum emission we present in this paper is primarily due to synchrotron emission and, at $353 \mathrm{GHz}$, dust emission. As polarized radiation propagates through the ionized ISM, depolarization effects reduce the polarized intensity by producing polarization vectors at different angles either along the line of sight or within the beam. The superposition of these vectors reduces the observed polarized intensity (Burn 1966; Tribble 1991; Sokoloff et al. 1998; Gaensler et al. 2001). Beam depolarization arises when different paths within the beam have different Faraday depths (see equation 1) and thus polarization angles. Foreground turbulent regions can lead to significant beam depolarization. Depth depolarization occurs when distant emission is Faraday rotated and cancels more local emission. Depth depolarization generally refers to an effect that occurs over a long path. Faraday screens can rotate background Faraday rotation and cause depolarization in a similar manner but over a very short path (Sun et al. 2007); we refer to this as Faraday screen depolarization. These three effects are results of Faraday rotation and are thus frequency dependent. Geometrical depolarization is the product of different orientations of the magnetic field at different distances along the line of sight resulting in the superposition of emission with different intrinsic polarization angles (Miville-Deschênes et al. 2008; Delabrouille et al. 2013). Geometrical depolarization is the most important depolarization mechanism at high frequencies. At $23 \mathrm{GHz}$, the change in polarization angle $\Delta \psi \approx 0.01 \mathrm{rad}$ (for $\phi=50 \mathrm{rad} \mathrm{m}^{-2}$ ), so Faraday rotation is negligible.

\section{KINEMATIC FEATURES AND DISTANCES}

Because all components of the ISM (atomic, molecular and ionized gas as well as the magnetic field) are inter-related, we examine the role of Galactic structure in producing both the ionized gas and the polarized emission from the Fan Region. In this section, we review our knowledge of Galactic structure and the distances to observed features in the second and third quadrants of the Galaxy.

Sightlines towards the Fan Region $\left(\ell \approx 130^{\circ}\right)$ pass through the Perseus and outer spiral arms. We illustrate this with an overhead view of the Galaxy in Fig. 1. The Perseus Arm, which Benjamin (2008) considers one of two 'major' spiral arms, is observed in ionized and neutral gas and star formation. It is about $2 \mathrm{kpc}$ away at a Galactocentric radius of $\approx 10 \mathrm{kpc}$, while the Outer Arm, likely a more minor arm with a concentration of gas but not of old stars,

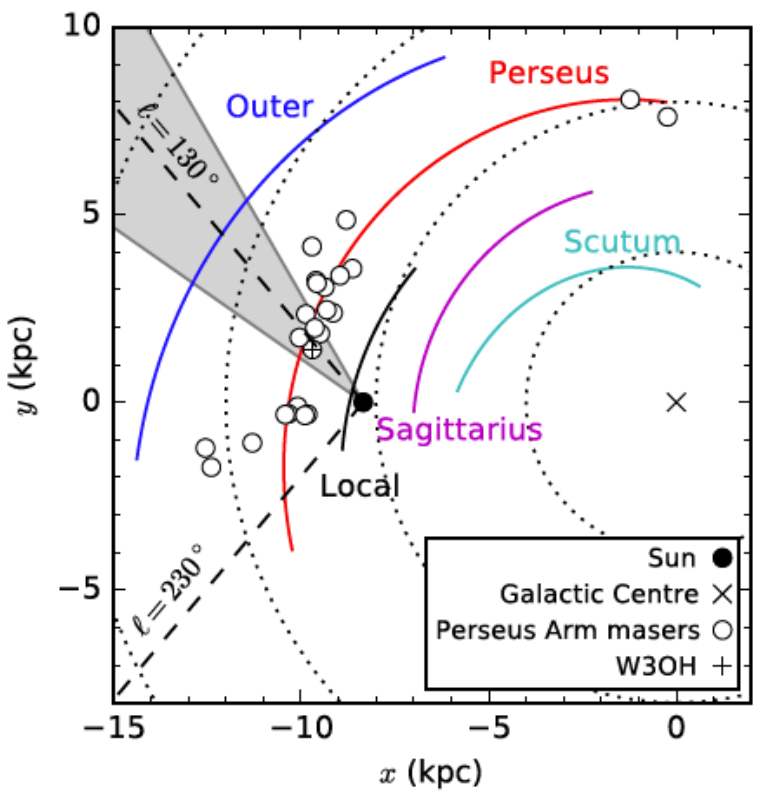

Figure 1. View of the Galaxy from above with spiral arms from Reid et al. (2014). The longitude range $120^{\circ}<\ell<145^{\circ}$, the region that contains the brightest emission from the Fan Region, is shaded grey.

is $\approx 6 \mathrm{kpc}$ away at a Galactocentric radius of $\approx 13 \mathrm{kpc}$ (e.g. Xu et al. 2006; Churchwell et al. 2009; Reid et al. 2014).

In the second quadrant, sightlines are closer to normal to these spiral arms than are similar sightlines in the third quadrant. As an example, we consider the two sightlines $50^{\circ}$ from the anticentre $\left(\ell=180^{\circ}\right), \ell=130^{\circ}$ and $\ell=230^{\circ}$. These are shown as dashed lines in Fig. 1. One might expect these sightlines to probe similar parts of the Galaxy. The angle between the $\ell=230^{\circ}$ sightline and the normal to the Perseus Arm is $\approx 45^{\circ}$, whereas the angle between the $\ell=130^{\circ}$ sightline and the Perseus Arm normal is $\approx 30^{\circ}$. For the outer arm, the offset between the $\ell=130^{\circ}$ sightline and the normal to the arm is $\approx 15^{\circ}$; the $\ell \approx 230^{\circ}$ sightline does not intersect any known Outer Arm material. If the Outer Arm continued at the same pitch angle, the $\ell=230^{\circ}$ sightline would encounter the Outer Arm much further out, $>20 \mathrm{kpc}$ from the Galactic Centre.

In Fig. 2, we plot longitude-velocity diagrams of $\mathrm{HI}$ and $\mathrm{H} \alpha$ to describe the structure of Galactic emission in this region. The Perseus Arm is the dominant feature, with the $\mathrm{H} \mathrm{I}$ and $\mathrm{H} \alpha$ emission brightest at $V_{\mathrm{LSR}} \approx-50 \mathrm{~km} \mathrm{~s}^{-1}$ in the mid-plane at $\ell=135^{\circ}$. There are kinematically distinct $\mathrm{H}$ I components at local and Perseus Arm velocities. Because the brightest $\mathrm{H} \alpha$ emission is concentrated in $\mathrm{H}_{\text {II }}$ regions, the $\mathrm{H} \alpha$ is patchier than the $\mathrm{H}$. Typical $\mathrm{H} \alpha$ linewidths are $\approx 30 \mathrm{~km} \mathrm{~s}^{-1}$, compared to $10 \mathrm{~km} \mathrm{~s}^{-1}$ for $\mathrm{H}_{\mathrm{I}}$, which also makes it more difficult to separate $\mathrm{H} \alpha$ components. However, the local and Perseus Arm $\mathrm{H} \alpha$ components are kinematically distinct at $150^{\circ}$ $\gtrsim \ell \gtrsim 130^{\circ}$ (Haffner, Reynolds \& Tufte 1999), and Fig. 2 shows distinct components at $V_{\mathrm{LSR}} \approx-50 \mathrm{~km} \mathrm{~s}^{-1}$ and $V_{\mathrm{LSR}} \approx-10 \mathrm{~km} \mathrm{~s}^{-1}$ extending to $\ell \approx 100^{\circ}$. We adopt $-75 \mathrm{~km} \mathrm{~s}^{-1}<V_{\mathrm{LSR}}<-30 \mathrm{~km} \mathrm{~s}^{-1}$ as the velocity range that defines the Perseus $\mathrm{Arm} \mathrm{H} \alpha$.

The distance to masers in the Perseus Arm ranges from 1.9 to $2.8 \mathrm{kpc}$ over $120^{\circ}<\ell<145^{\circ}$ with a line-of-sight depth of $0.38 \mathrm{kpc}$ from parallax measurements (Reid et al. 2009, 2014, and references therein). We show the longitudes and velocities of the masers Reid et al. used to determine this distance in Fig. 2. The maser positions and velocities trace both the $\mathrm{H} \alpha$ and $\mathrm{HI}_{\mathrm{I}}$ emission. We also plot lines showing the longitude and velocity of the Perseus and Outer 

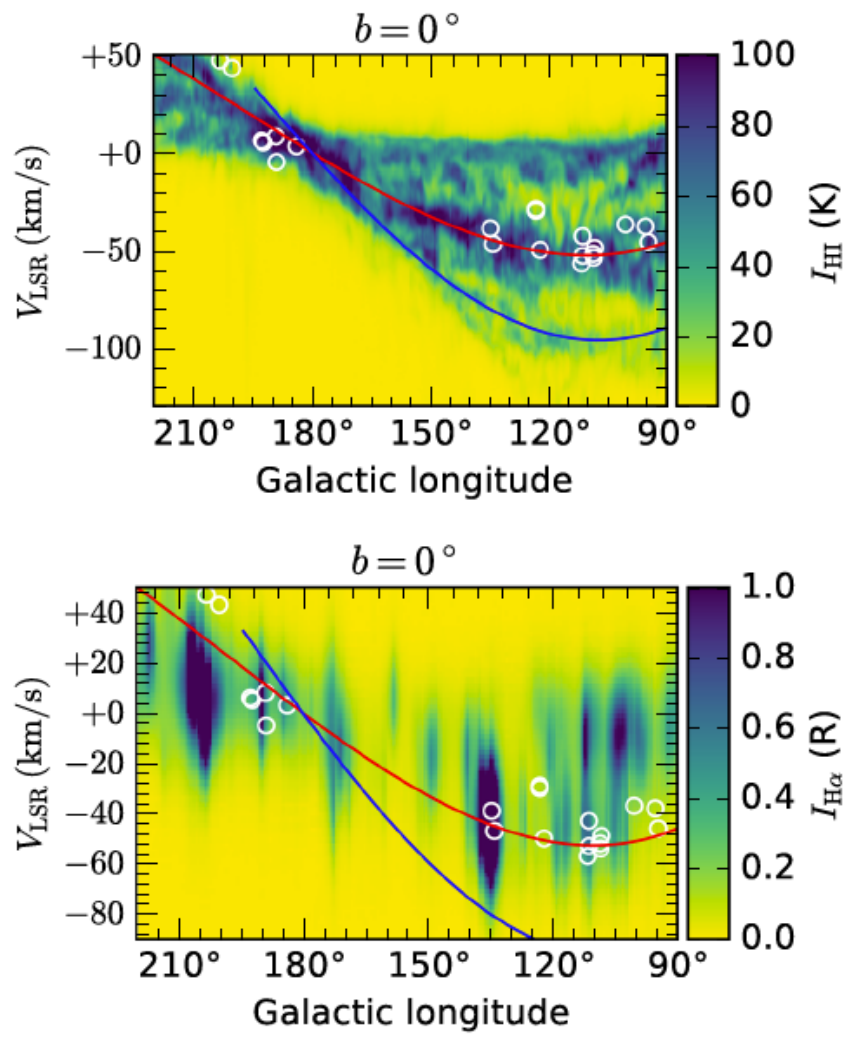

Figure 2. Longitude-velocity diagrams of $\mathrm{H}_{\mathrm{I}}$ from LAB (top) and $\mathrm{H} \alpha$ from WHAM (bottom). White circles show the longitudes and velocities of masers in the Perseus Arm from Reid et al. (2014), as in Fig. 1. Red and blue lines show the Perseus and Outer arms, respectively (see the text).

arms with velocities derived using the linear function defining the Galactic rotation speed from Reid et al. (2014, their Bayesian fit D1). Because kinematic distances in the second quadrant overestimate the true source distance measured by parallax (Foster \& MacWilliams 2006; Reid et al. 2009) and the purpose of these lines is simply to guide the eye, we multiplied the derived velocities for the arms by 1.5 and 1.3 , respectively, to match the lines to the kinematic features.

The latitude-velocity diagram in Fig. 3 shows the warp in the Fan Region. There is evidence of a warp in the further reaches of the Perseus Arm: the bright emission at $V_{\mathrm{LSR}} \approx-45 \mathrm{~km} \mathrm{~s}^{-1}$ is centred at $b=0^{\circ}$ but gas at more negative velocities predominantly lies above $b=0^{\circ}$. At $V_{\mathrm{LSR}} \approx-75 \mathrm{~km} \mathrm{~s}^{-1}$, the warp is evident at $b \approx+3^{\circ}$. This is the Outer Arm, at a distance of $\approx 6 \mathrm{kpc}$ (Hachisuka et al. 2009; Reid et al. 2014). At $V_{\mathrm{LSR}} \approx-100 \mathrm{~km} \mathrm{~s}^{-1}$, the $\mathrm{H}$ I emission from the warp is brightest at $b \approx+6^{\circ}$, while it extends to $b=+8^{\circ}$ at $V_{\mathrm{LSR}}$ $\approx-115 \mathrm{~km} \mathrm{~s}^{-1}$. In this region, there is $\mathrm{H} \mathrm{I}$ emission near $b=0^{\circ}$ out to large velocities in addition to the warped emission.

In Fig. 3, we also show a fit to the warp along a sightline in the Fan Region, calculating following Kalberla et al. (2007, with data provided by Kalberla, private communication). In this fit, the emission is centred near $b=+3^{\circ}$ from $1 \mathrm{kpc} \lesssim D \lesssim 5 \mathrm{kpc}$, near $b=+5.5$ from $8 \mathrm{kpc} \lesssim D \lesssim 15 \mathrm{kpc}$, and near $b=+9^{\circ}$ over $D \gtrsim$ $20 \mathrm{kpc}$. The emission at Perseus Arm velocities around $\ell=130^{\circ}$ is centred near $b=+1^{\circ}$, while emission at Outer Arm velocities in this region is centred near $b=+3^{\circ}$ at a distance of $6 \mathrm{kpc}$ (Hachisuka et al. 2009; Reid et al. 2014).

There is no clear evidence of a warp in $\mathrm{H} \alpha$ emission, likely due to four factors: (1) the broader linewidths of $\mathrm{H} \alpha\left(\approx 30-40 \mathrm{~km} \mathrm{~s}^{-1}\right.$,
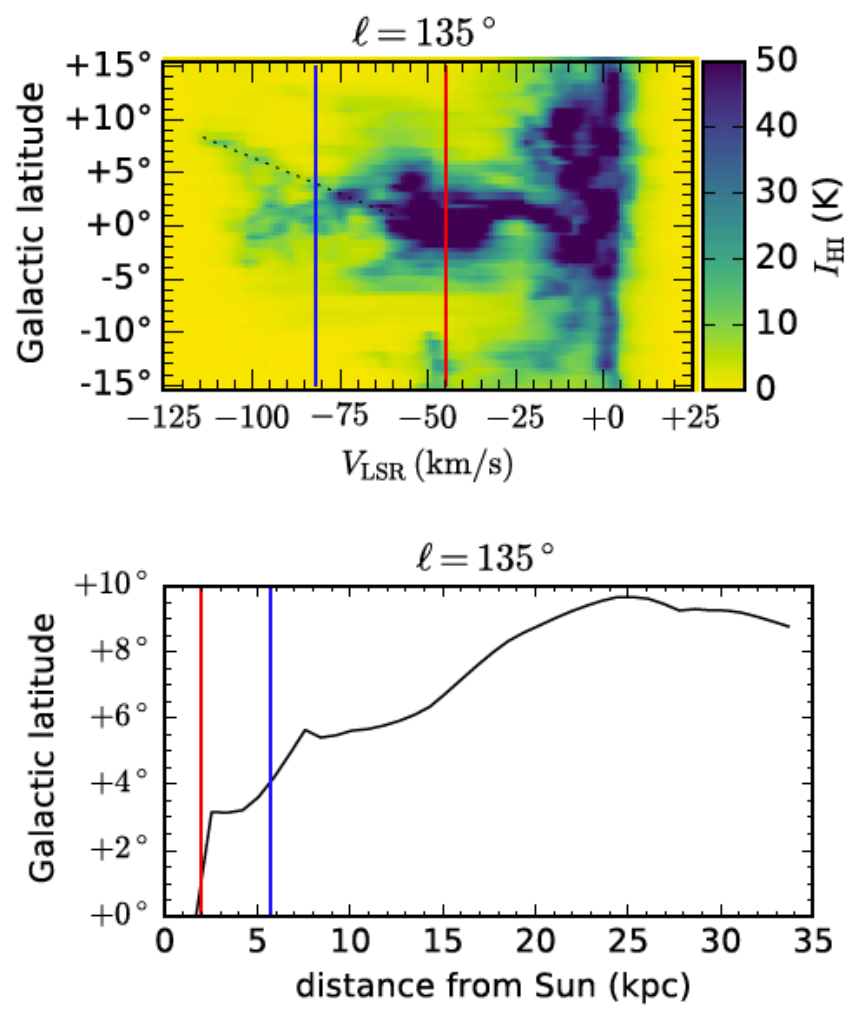

Figure 3. Top: latitude-velocity diagram of $\mathrm{H}_{\mathrm{I}}$ from LAB. The velocities of the Perseus (red) and Outer (blue) arms at this longitude as shown in Fig. 2 are shown. A dotted line indicates the approximate position of the warp emission. Bottom: latitude of the mid-plane of the warped Galaxy as a function of distance from the Sun calculated using the fit to the LAB data determined by Kalberla et al. (2007). For a discussion of the considerable uncertainties in this plot, see their paper. Distances to the arms at $\ell=135^{\circ}$ from the fit to maser distances (Reid et al. 2014) are shown as an indication, but the systematic uncertainties in the kinematic distances used to determine the latitude of the warp are large.

evident in Fig. 2) make it difficult to separate the Outer Arm from the Perseus Arm in $\mathrm{H} \alpha$; (2) extinction makes distant $\mathrm{H} \alpha$ more difficult to observe (Madsen \& Reynolds 2005); (3) the scaleheight of the warm ionized medium (WIM) is large enough (1-1.4 kpc; Haffner et al. 1999; Gaensler et al. 2008; Savage \& Wakker 2009; Schnitzeler 2012) that a $3^{\circ}$ offset of the mid-plane is a relatively small effect and (4) the star formation rate in the Outer Arm may be lower because star formation rates are typically lower further from the Galactic Centre (Kennicutt \& Evans 2012), producing a lower ionizing flux and thus less $\mathrm{H} \alpha$ emission.

\section{RESULTS}

\subsection{Morphology of polarized emission}

In Fig. 4, we show the Fan Region in its Galactic context. The allsky images are centred on the second quadrant $\left(90^{\circ} \lesssim \ell \lesssim 180^{\circ}\right)$, where the Fan Region is located. The Fan Region is identified by the bright signal in polarized intensity at $1.5 \mathrm{GHz}\left(L_{1.5}\right), 23 \mathrm{GHz}$ $\left(L_{23}\right)$ and $353 \mathrm{GHz}\left(L_{353}\right)$. At $1.5 \mathrm{GHz}$, the polarized emission is centred above the plane, with $L_{1.5} \gtrsim 0.1 \mathrm{~K}$ (green in Fig. 4) emission extending over $-12^{\circ} \lesssim b \lesssim+25^{\circ}, 90^{\circ} \lesssim \ell \lesssim 180^{\circ}$. The $23 \mathrm{GHz}$ and $353 \mathrm{GHz}$ emissions are roughly similar, in both cases with emission centred at $b \approx+4^{\circ}$. 

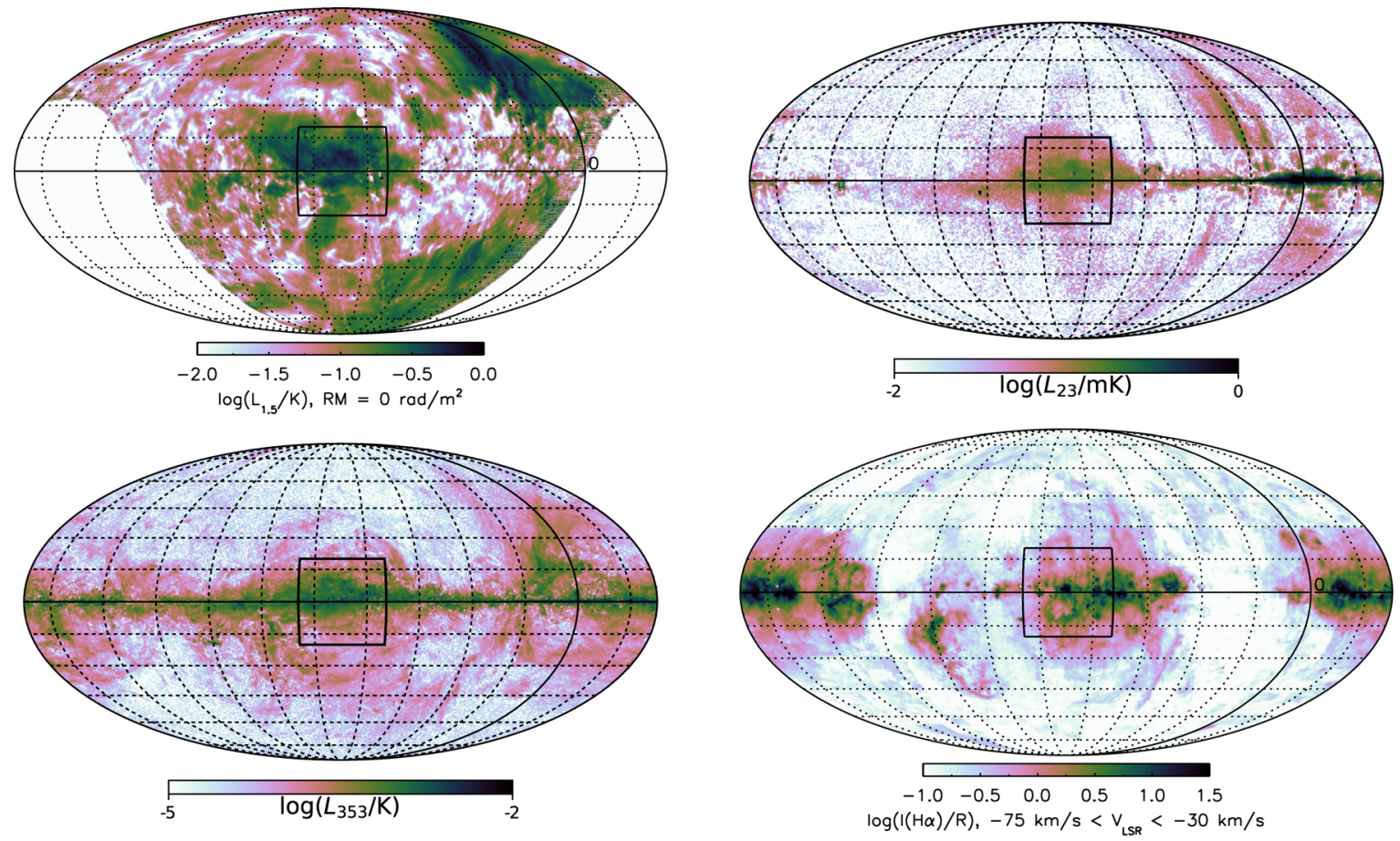

Figure 4. All-sky images centred on $\ell=135^{\circ}$ and $b=0^{\circ}$ in a Mollweide projection with grid lines every $\Delta \ell=30^{\circ}, \Delta b=15^{\circ}$. Solid lines show $\ell=0^{\circ}$ and $b=0^{\circ}$. The intensity scales in each image are logarithmic. Top left: GMIMS-HBN $1.5 \mathrm{GHz}$ polarized intensity at Faraday depth 0 rad $\mathrm{m}^{-2}$. Top right: WMAP $23 \mathrm{GHz}$ linearly polarized intensity (Bennett et al. 2013). Bottom left: Planck $353 \mathrm{GHz}$ linearly polarized intensity from the 2015 data release (Planck Collaboration I 2016) smoothed with a 20 arcmin FWHM Gaussian beam. Bottom right: WHAM-SS H $\alpha$ emission (Haffner et al. 2003, 2010) integrated over $-75 \mathrm{~km} \mathrm{~s}^{-1}<V_{\mathrm{LSR}}<-30 \mathrm{~km} \mathrm{~s}^{-1}$, isolating emission from the Perseus Arm and beyond. Boxes indicate the corners of the region shown in Fig. 5.

The brightest emission, $L_{1.5} \gtrsim 0.3 \mathrm{~K}$, is mostly in a smaller region, $120^{\circ}<\ell<150^{\circ}$ and $-6^{\circ}<b<+12^{\circ}$. We show maps of polarized intensity in this region from $408 \mathrm{MHz}$ to $23 \mathrm{GHz}$ in Fig. 5. The morphology is qualitatively different at $v \lesssim 600 \mathrm{MHz}$ and $v \gtrsim$ $1 \mathrm{GHz}$. At high frequencies, the emission fills most of this region. At $23 \mathrm{GHz}$, the emission is bright both at $|b| \lesssim 2^{\circ}$ and above the plane up to $+10^{\circ}$. In contrast, at $1.5 \mathrm{GHz}$ and all lower frequencies, the emission is much fainter in the plane $\left(|b| \lesssim 2^{\circ}\right)$ than at $b \approx$ $+8^{\circ}$. However, the bright emission is similar in morphology at 23 and $1.5 \mathrm{GHz}$ at $b \gtrsim+3^{\circ}$. At both frequencies, the bright emission follows an arc from around $\left(134^{\circ},+8^{\circ}\right)$ to $\left(128^{\circ},+3^{\circ}\right)$, emphasized by the yellow contours in Fig. 5 .

There is no obvious feature corresponding to the Fan Region in total intensity at $1.4 \mathrm{GHz}$. We show the polarization fraction at $\sim 1.5 \mathrm{GHz}$ in Fig. 6 . The structure of the polarization fraction image closely traces that of the polarized intensity image (Fig. 5b) because the Stokes $I$ emission is much more uniform than the polarized intensity. The polarization fraction is highest, $\approx 40$ per cent, in the region where $L_{1.5}$ is brightest, in the arc that peaks at $\left(134^{\circ},+8^{\circ}\right)$.

There is a patch where the polarized intensity at $1.5 \mathrm{GHz}$ is lower, centred at $\left(134.5,+4^{\circ}\right)$ and $\approx 5^{\circ}$ in diameter; this area, shown with black circles in Figs 5-7 as well as Figs 11-12 below, is a major focus of this paper. The reduced $L_{1.5}$ cannot be entirely due to Faraday depolarization because it is also seen to some extent in $L_{23}$. However, the $1.5 \mathrm{GHz}$ fractional polarization in Fig. 6 is lower in that patch $(\approx 30$ percent $)$ than in the surrounding Fan Region $(\approx 40$ per cent $)$.

This reduction in polarized intensity is not seen in the Dwingeloo $1411 \mathrm{MHz}$ data (Brouw \& Spoelstra 1976; Carretti et al. 2005). That survey is sparsely sampled, and by checking the measured data points we have verified that there was no measurement near $\left(134.5^{\circ}\right.$, $+4^{\circ}$ ): the $1411 \mathrm{MHz}$ survey could not have detected this feature. A similar check of the $820 \mathrm{MHz}$ observation points shows that there was a measurement close to this position, and the interpolated image at $820 \mathrm{MHz}$ does indeed show a decline in polarized intensity at this position (Fig. 5c).

Somewhat puzzling is the fact that the depression in polarized intensity is not seen in the Wolleben et al. (2006) data. That survey was made using drift scans, and a check showed that drift scans were made across this region at full sampling; however, no cross scans in declination were made. We regard the GMIMS data as more reliable. Scanning was in two directions, fully Nyquist sampled, and the area of interest was crossed by many scans. The intersecting scans were reconciled by the basketweaving technique. Inspection of the GMIMS data cube shows that the depression in polarized intensity is seen at every frequency across the GMIMS band as well as in the image after RM synthesis.

At the lowest frequencies, the polarized emission is brightest in a ring centred at $\left(137^{\circ},+8^{\circ}\right)$, shown with blue circles in Fig. 5. At $150-350 \mathrm{MHz}$, this ring is the dominant feature identifiable in the Fan Region, with a diameter of about $7^{\circ}$ in Westerbork Synthesis Radio Telescope data. Various authors have used Westerbork data to describe the ring as a relic Strömgren sphere at a distance of $\approx 200$ pc (Iacobelli et al. 2013a) and as a depolarization artefact of a uniform Faraday depth distribution (Haverkorn, Katgert \& de Bruyn 2003). The Westerbork data use aperture synthesis and thus are not sensitive to features $\gtrsim 10^{\circ}$ in size (Bernardi et al. 2009), whereas the Brouw \& Spoelstra (1976) data are single-antenna and thus should be sensitive to emission on all resolved scales. The ring is also the dominant feature at frequencies up to $610 \mathrm{MHz}$ (see Fig. 5). Its diameter is larger at higher frequencies, and it also becomes less clearly defined as a circular feature. At $v \gtrsim 1.5 \mathrm{GHz}$, 

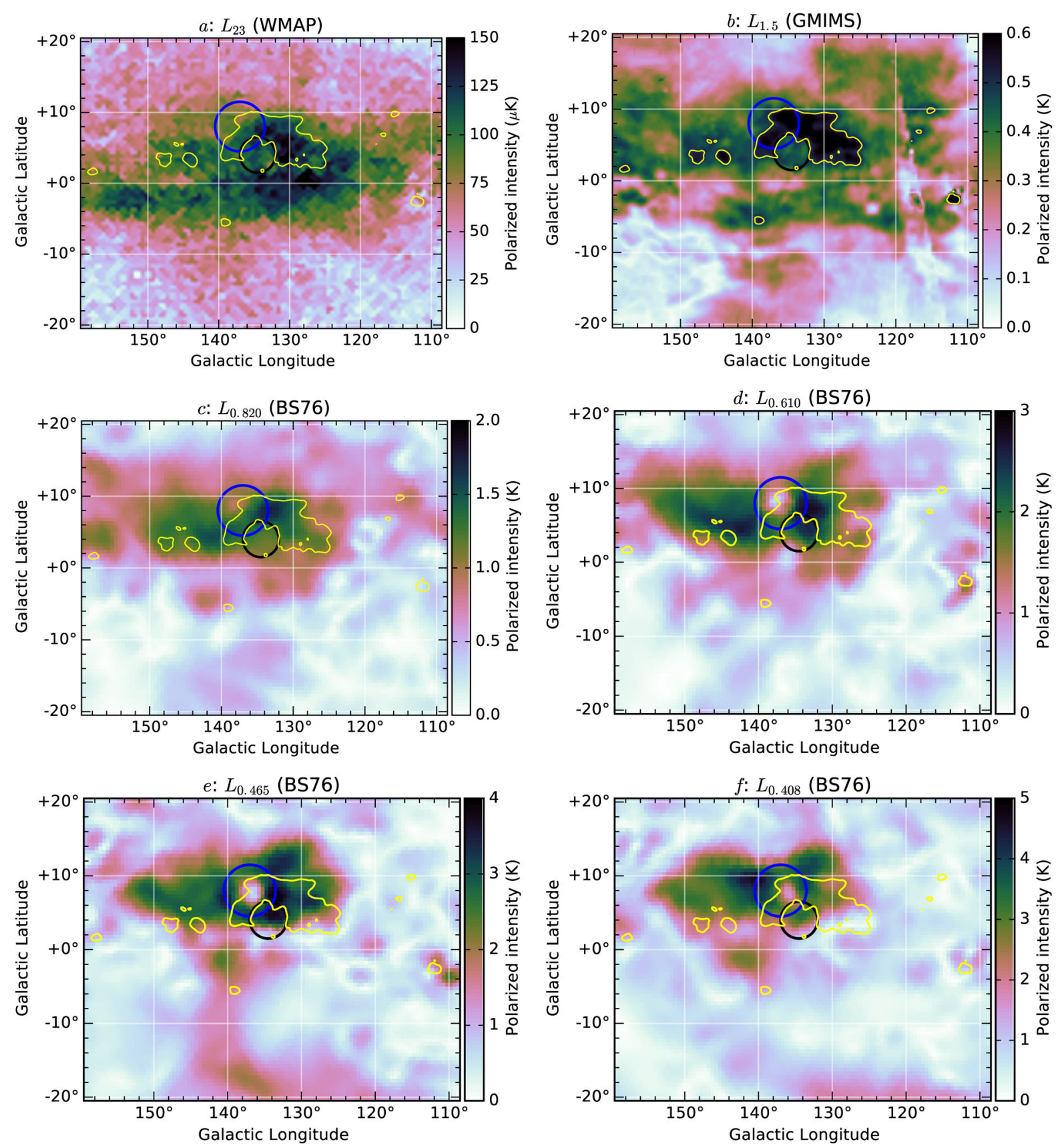

Figure 5. Images of polarized intensity at $23 \mathrm{GHz}$ (WMAP), $1.5 \mathrm{GHz}$ (GMIMS) and 408-820 MHz (Brouw \& Spoelstra 1976; Carretti et al. 2005). Yellow contours show GMIMS data $\left(L_{1.5}\right)$ at an antenna temperature of $0.5 \mathrm{~K}$. Black and blue circles show the features at $\left(134.5^{\circ},+4^{\circ}\right)$ and $\left(137^{\circ},+8^{\circ}\right)$ discussed in the text. A small artefact remains in the GMIMS $L_{1.5}$ image at $0 \mathrm{~h}$ right ascension (the diagonal stripe around $\ell=115^{\circ}$ in panel $b$ ).

the ring is not apparent. At $820 \mathrm{MHz}$, both the ring and the broader, high-frequency feature are evident.

\subsection{Morphological comparison of $\mathrm{H} \alpha$ and $1-23 \mathrm{GHz}$ polarized emission}

The lower right panel of Fig. 4 shows $\mathrm{H} \alpha$ emission with a velocity criterion $\left(-75 \mathrm{~km} \mathrm{~s}^{-1}<V_{\mathrm{LSR}}<-30 \mathrm{~km} \mathrm{~s}^{-1}\right)$ that excludes local gas but includes emission from the Perseus Arm and more distant gas (Haffner et al. 1999; Madsen, Reynolds \& Haffner 2006). We compare $L_{1.5}$ with $\mathrm{H} \alpha$ at the Perseus Arm velocity as well as at local velocities and integrated over all velocities in Fig. 7. As in the polarized continuum maps, the $\mathrm{H} \alpha$ emission is brightest around $100^{\circ}$ $\lesssim \ell \lesssim 150^{\circ}$, although the $\mathrm{H} \alpha$ emission is centred at somewhat lower latitudes $\left(b \approx 0^{\circ}\right)$ and longitudes than the polarized continuum. The highest $1.5 \mathrm{GHz}$ polarized intensity, $L_{1.5} \approx 0.65 \mathrm{~K}$, is in a region [near $\left(134^{\circ},+8^{\circ}\right)$ ] with little $\mathrm{H} \alpha$ emission at any velocity. The region with depressed $L_{1.5}$ around $\left(134.5^{\circ},+4^{\circ}\right.$ ) (which we introduced in Section 4.1) corresponds with increased $\mathrm{H} \alpha$ intensity at Perseus Arm velocities. The white $\left(I_{\mathrm{H} \alpha}=2.0 \mathrm{R}\right)$ contour $^{3}$ in Fig. 7 (c) traces the lower envelope of the bright $\left[L_{1.5} \gtrsim 0.5 \mathrm{~K}\right.$; dark

\footnotetext{
${ }^{3} 1$ Rayleigh $(\mathrm{R})=10^{6} /(4 \pi)$ photons $\mathrm{cm}^{-2} \mathrm{~s}^{-1} \mathrm{sr}^{-1}$.
} 


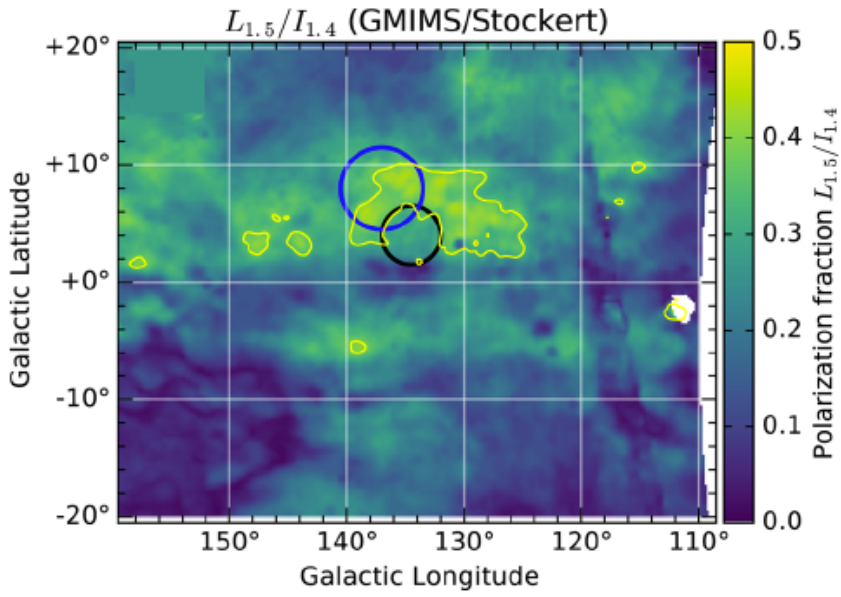

Figure 6. Polarization fraction from comparing the GMIMS data to the Stockert $1.4 \mathrm{GHz}$ Stokes $I$ data (Reich 1982). Yellow contours show $L_{1.5}=0.5 \mathrm{~K}$, as in Fig. 5 .

blue in Figs 7(a)-(c)] polarized emission, suggesting qualitatively that the polarized intensity in this region is anticorrelated with the Perseus Arm $\mathrm{H} \alpha$ intensity.

We quantify the relationship between $L_{1.5}$ and $I_{\mathrm{H} \alpha}$ from the Perseus Arm in the scatter plot in Fig. 8. We chose a narrow latitude range to minimize the impact of the latitude dependence of $I_{\mathrm{H} \alpha}$ on the scatter plot. The latitude range $+3^{\circ}<b<+6^{\circ}$ cuts through the

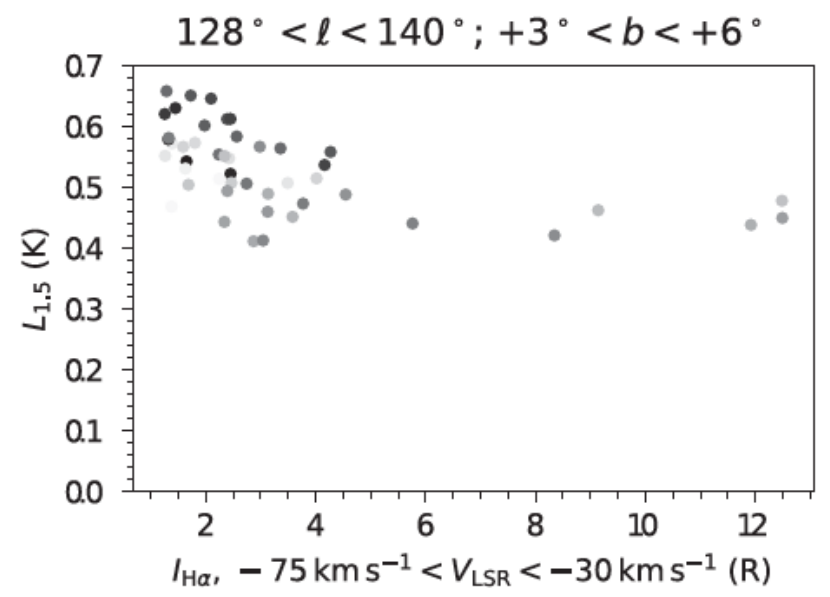

Figure 8. Polarized intensity $\left(L_{1.5}\right)$ as a function of integrated $\mathrm{H} \alpha$ intensity from the Perseus Arm $\left(-75 \mathrm{~km} \mathrm{~s}^{-1}<V_{\mathrm{LSR}}<-30 \mathrm{~km} \mathrm{~s}^{-1}\right)$. Each point denotes $I_{\mathrm{H} \alpha}$ from a single WHAM beam compared to the average GMIMS $L_{1.5}$ within the beam in between the lines shown in Fig. 7. Points are colour coded for Galactic longitude, with the lightest points at $\ell=140^{\circ}$ and black points at $\ell=128^{\circ}$.

region of reduced $L_{1.5}$ around $\left(134.5,+4^{\circ}\right)$ we discussed in Section 4.1 . We chose the longitude range $128^{\circ}<\ell<140^{\circ}$ to encompass the bright emission from the Fan Region as seen at $1.5 \mathrm{GHz}$, $23 \mathrm{GHz}$ and $353 \mathrm{GHz}$ (see Figs 4 and 5). In the six sightlines in the plot with $I_{\mathrm{H} \alpha}>5 \mathrm{R}$, the polarized intensity is very consistent,
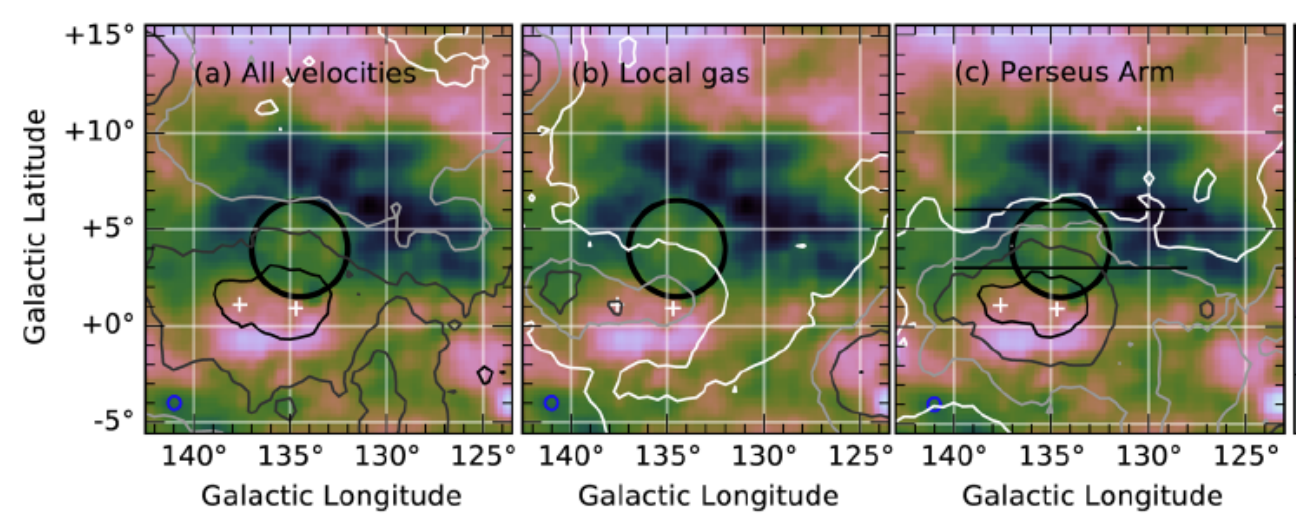

0.7
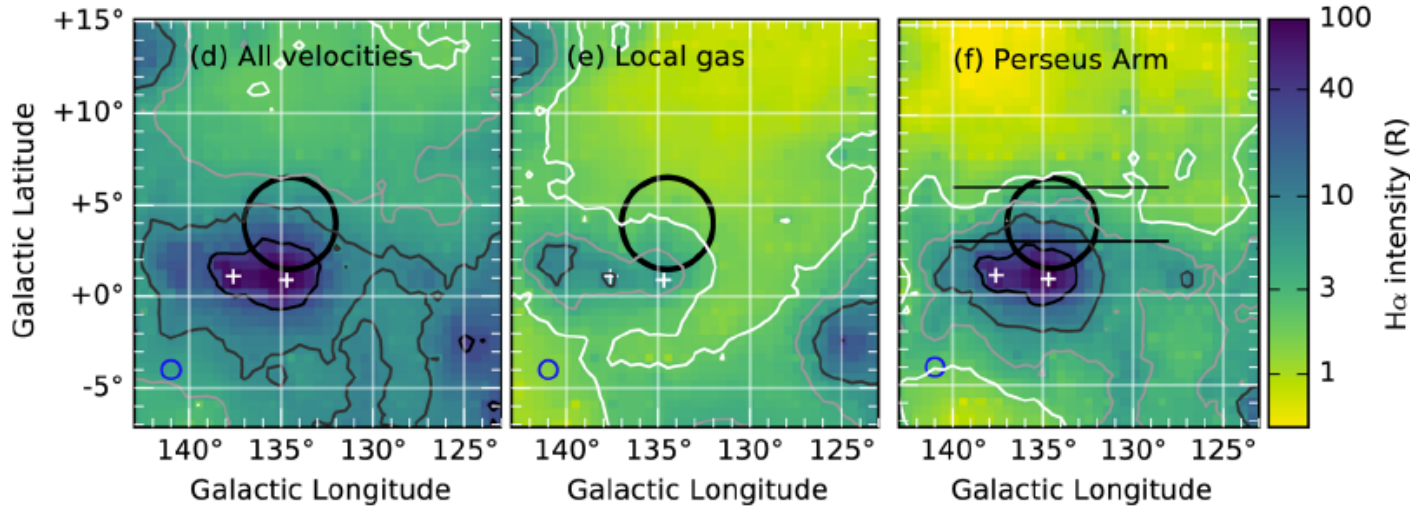

Figure 7. Top row: $1.5 \mathrm{GHz}$ polarized continuum emission from GMIMS (as in Fig. 5b); we show the same map in all three columns. Bottom row: WHAM-SS $\mathrm{H} \alpha$ maps in three velocity ranges. Left: all velocities with significant emission $\left(-75 \mathrm{~km} \mathrm{~s}^{-1}<V_{\mathrm{LSR}}<+15 \mathrm{~km} \mathrm{~s}^{-1}\right)$. Middle: local velocities $\left(\left|V_{\mathrm{LSR}}\right|<15 \mathrm{~km} \mathrm{~s}^{-1}\right)$. Right: Perseus Arm velocities $\left(-75 \mathrm{~km} \mathrm{~s}^{-1}<V_{\mathrm{LSR}}<-30 \mathrm{~km} \mathrm{~s}^{-1}\right)$. Corresponding $\mathrm{H} \alpha$ contours at intensities of 2 (white), 4 (light grey), 8 (dark grey) and $32 \mathrm{R}$ (black) are shown in both rows. White + signs show the centres of W4 and W5. Horizontal lines in panels $c$ and $f$ show the region used to calculate Fig. 8. Black circles are as in Fig. 5. $1.5 \mathrm{GHz}$ data are shown with a linear scale; $\mathrm{H} \alpha$ data are shown with a logarithmic scale. The GMIMS (top) and WHAM (bottom) beams are shown with blue circles in the lower left corner. 


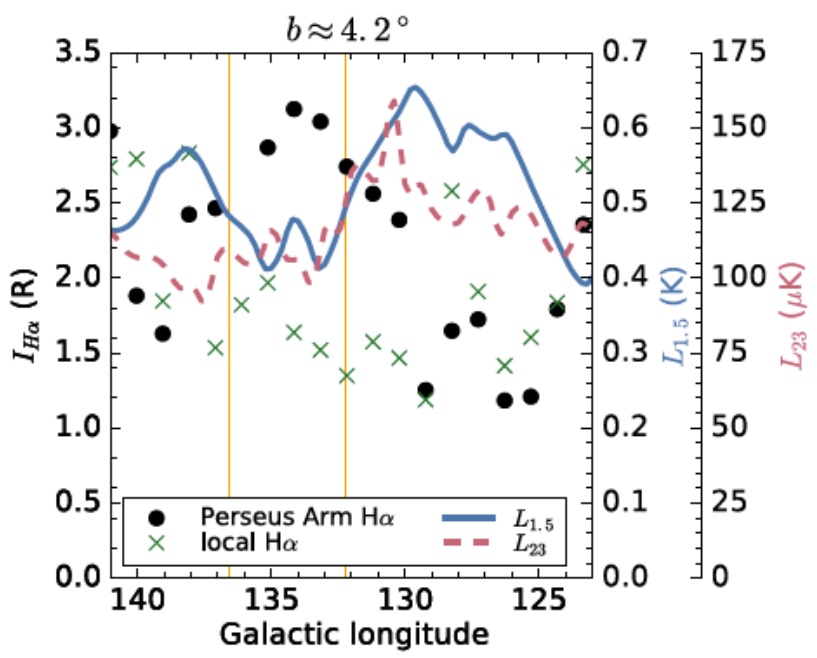

Figure 9. Polarized intensity $\left(L_{1.5}\right.$ and $\left.L_{23}\right), \mathrm{H} \alpha$ intensity at Perseus Arm velocities $\left(-75 \mathrm{~km} \mathrm{~s}^{-1}<V_{\mathrm{LSR}}<-30 \mathrm{~km} \mathrm{~s}^{-1}\right)$ and $\mathrm{H} \alpha$ intensity at local velocities $\left(\left|V_{\mathrm{LSR}}\right|<15 \mathrm{~km} \mathrm{~s}^{-1}\right)$ as a function of Galactic longitude in the range $+4^{\circ} \leq b \leq+5^{\circ}$. The H $\alpha$ points shown are from individual WHAM beams at $b=+4.2^{\circ}$ because the WHAM survey is not Nyquist sampled. Vertical orange lines show the outer boundaries of the W4 superbubble walls from $I_{4.8}$ data (see Fig. 12 below).

$\left\langle L_{1.5}\right\rangle=0.44 \mathrm{~K}$ with standard deviation $\sigma_{L 1.5}=0.02 \mathrm{~K}$. In sightlines with $I_{\mathrm{H} \alpha}<3 \mathrm{R}$, there is a wider range of polarized intensities, typically $\approx 30$ per cent higher than in the $\mathrm{H} \alpha$-bright sightlines. The anticorrelation of $L_{1.5}$ with $I_{\mathrm{H} \alpha}$ is most likely due to depolarization, not an intrinsic absence of polarized emission along these sightlines. We expect depolarization due to all gas in front of the emission, so correlating structures in depolarization with $\mathrm{H} \alpha$ emission at an estimated distance measures the minimum distance to the emitting gas. The $\mathrm{H} \alpha$ emission from the Perseus Arm [Figs 7(c) and (f)] is brightest in a roughly circular region centred on the W3/W4/W5 complex of $\mathrm{H}$ II regions. (W4 is shown with $\mathrm{a}+\operatorname{sign}$ in Fig. 7.) From the optical line ratios, this bright $\mathrm{H} \alpha$ emission is more typical of $\mathrm{O}$ star $\mathrm{H}_{\text {II }}$ regions than of the diffuse WIM (Madsen et al. 2006), indicating that the gas is primarily photoionized by $\mathrm{O}$ stars in those $\mathrm{H}$ II regions. The arched shape of the $1.5 \mathrm{GHz}$ emission is matched by an arch in the Perseus Arm H $\alpha$ contour at $\left(134^{\circ},+7^{\circ}\right)$ in Fig. 7(c). This fainter $\mathrm{H} \alpha$ from the Perseus Arm, which is spatially coincident with the high polarized intensity from the Fan Region, has optical line ratios (especially $[\mathrm{N}$ II] $\lambda 6584 / \mathrm{H} \alpha$ ), which are higher and thus more WIM-like than the $\mathrm{H}$ II region emission (see fig. 11 of Madsen et al. 2006).

We have not applied an extinction correction to the $\mathrm{H} \alpha$ data. From a comparison of $\mathrm{H} \alpha$ and $\mathrm{H} \beta$ observations, there are $A_{V}=3.13 \pm 0.2$ mag of extinction towards the $\mathrm{W} 4 \mathrm{H}$ II region at $\mathrm{H} \alpha$ at $\left(134.7^{\circ},+0.9^{\circ}\right)$, the brightest sightline in this region in $\mathrm{H} \alpha$ (Madsen et al. 2006). Robust extinction measurements are only available for a small number of individual sightlines, so a systematic extinction correction is difficult. We expect that the dust column and thus the extinction is highest in sightlines that are brightest in $\mathrm{H} \alpha$, so we expect that the main impact of an extinction correction would be to increase the contrast between the faint sightlines and the bright ones. This would not change our interpretation of Figs 7 and 8: the sightlines with high $L_{1.5}$ would still be the sightlines with the lowest $I_{\mathrm{H} \alpha}$.

In Fig. 9, we show a slice of 1.5 and $23 \mathrm{GHz}$ polarized intensities and the Perseus Arm $\mathrm{H} \alpha$ intensity. In this slice, the $\mathrm{H} \alpha$ intensity peaks at $\ell=134^{\circ}$, the location of the minimum in $L_{1.5}$. At $1.5 \mathrm{GHz}$,

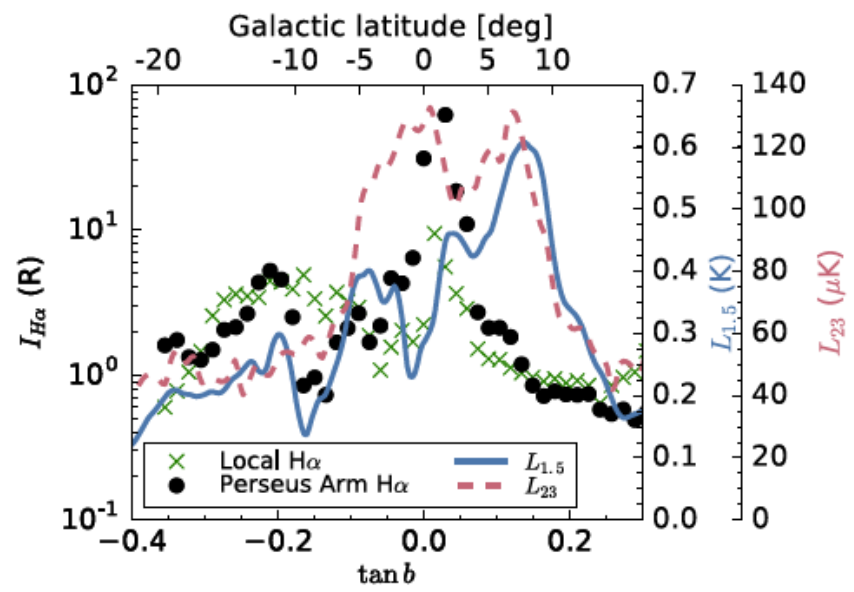

Figure 10. Vertical profiles of polarized intensity and $\mathrm{H} \alpha$ intensity (like Fig. 9), averaged over $133^{\circ}<\ell<136^{\circ}$. Note that the $\mathrm{H} \alpha$ is shown on a logarithmic scale, while the polarized intensity is shown on linear scales.

the polarized intensity peaks at $\ell \approx 130^{\circ}$ and $138^{\circ}$, areas of relatively low $\mathrm{H} \alpha$ intensity, and has a local minimum near $\ell=134^{\circ}$. A similar trend is evident at $820 \mathrm{MHz}$ (not shown) but not at lower radio frequencies. This trend is also evident at $23 \mathrm{GHz}$, although the local maximum at $\ell=138^{\circ}$ is not present. The figure also shows the local $\mathrm{H} \alpha$ emission, which does not have a discernible peak either correlated or anticorrelated with the polarized intensity. This indicates that the depolarization is due to $\mathrm{H} \alpha$-emitting gas at the velocity of the Perseus Arm.

The vertical profile in Fig. 10 shows that the $1.5 \mathrm{GHz}$ synchrotron intensity is highest at $b \approx+8^{\circ}$, with no sign of a comparable enhancement at $b \approx-8^{\circ}$. The $23 \mathrm{GHz}$ polarized emission is relatively bright over the range $-5^{\circ} \lesssim b \lesssim+10^{\circ}$. The upper envelopes of $L_{1.5}$ and $L_{23}$ around $b=+10^{\circ}$ are similar. The $1.5 \mathrm{GHz}$ intensity is much fainter at $b<+5^{\circ}$, whereas the $23 \mathrm{GHz}$ intensity is comparable at $b \approx 0^{\circ}$ and $b=+8^{\circ}$. We interpret this as the result of beam depolarization; $I_{\mathrm{H} \alpha} \approx 3-4 \mathrm{R}$ at $b=-5^{\circ}$, compared to $\approx 1 \mathrm{R}$ at corresponding latitudes above the plane. In our picture, the $23 \mathrm{GHz}$ and $1.5 \mathrm{GHz}$ emissions each originates in the same volume. The $23 \mathrm{GHz}$ polarized intensity traces the full extent of the synchrotron-emitting region without Faraday depolarization, while some of the $1.5 \mathrm{GHz}$ emission is Faraday depolarized.

\subsection{Using CGPS data to locate the Fan Region}

High-resolution data are less subject to beam depolarization than the GMIMS data. We use polarization data from the CGPS to obtain information relevant to the Fan Region at low latitudes, shown in Fig. 11. In the vicinity of the Fan Region, the angular resolution is close to 1 arcmin. We also refer to figs 5-7 of Landecker et al. (2010), which show images of total intensity and polarized intensity from the CGPS over $66^{\circ}<\ell<186^{\circ}$.

The images show many areas of emission that is structured on small angular scales. Virtually all of this emission is unrelated to objects seen in total intensity: these are typical Faraday screen structures.

The polarized intensity on and around W4 at $(134.7,+0.9)$ and W5 at $(137.6,+1.1)$, traced by $I_{1.4}$ (white) and $\mathrm{H} \alpha$ (black) contours in Fig. 11, stands out from the highly structured surroundings. Towards these two $\mathrm{H}$ II regions there is a marked absence of small-scale structure. Along the southern perimeter of these $\mathrm{H}$ II 


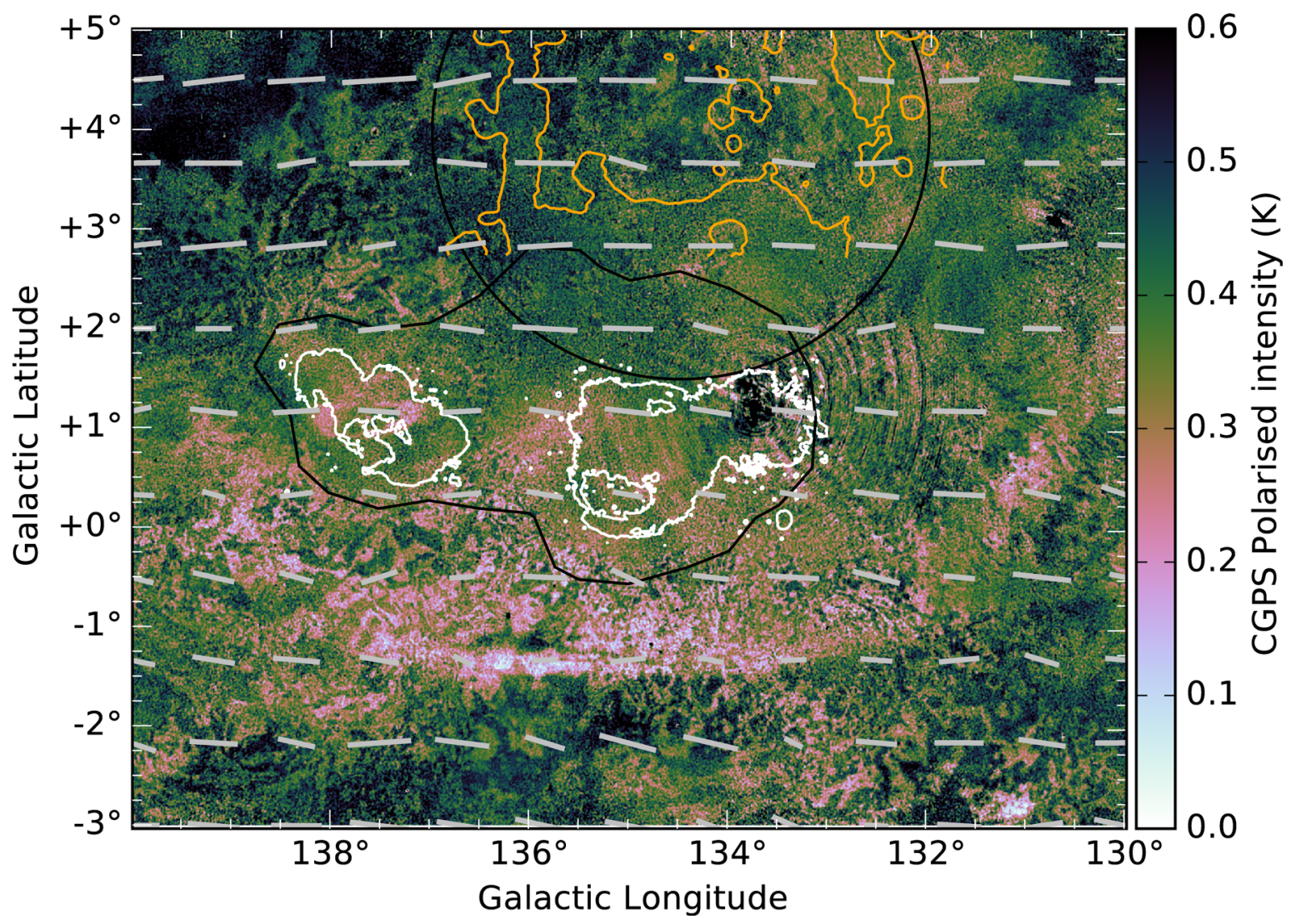

Figure 11. Polarized intensity at $1.4 \mathrm{GHz}$ from the CGPS survey (Landecker et al. 2010). CGPS magnetic field vectors are overplotted, and white contours show the CGPS total intensity $I_{1.4}$ at $7.5 \mathrm{~K}$. Orange contours show $4.8 \mathrm{GHz}$ total power in the W4 superbubble from Gao et al. (2010, 2015). The black contour shows the WHAM-SS H $\alpha$ intensity at Perseus Arm velocities at $32 \mathrm{R}$ as in Figs 7(c) and (f). Note that the WHAM-SS data have a $1^{\circ}$ resolution and were obtained on a $1^{\circ}$ grid, so structure details on smaller scales are unreliable. The black circle is as in Figs 5-7.

regions, but offset from the edge, there is an abrupt transition to highly structured polarized emission. This can also be seen clearly in figs 7 and 8 of West et al. (2007). Gray et al. (1999) showed that this transition below W4 is related to the $\mathrm{H}$ II region (and that is why the transition mirrors its shape): there is an extended, low-density ionized halo that extends beyond the boundaries defined by $1.5 \mathrm{GHz}$ continuum imaging in total intensity; this halo is evident with the WHAM data we show in Figs 7 and 11 as well as in $2.7 \mathrm{GHz}$ total intensity in fig. 7 of Gray et al. (1999). The gradient of Faraday rotation in that envelope is evidently sufficient to cause beam depolarization. The density of ionized gas drops off with distance beyond the $\mathrm{H}$ II region until the gradient of rotation is no longer sufficient to cause beam depolarization, and the small-scale structure re-appears.

Our conclusion is that most, and probably all, of this highly structured emission originates in the Perseus Arm, possibly in the vicinity of W4 and W5 which are on the near surface of the Arm but possibly further inside the Arm. The smoother emission seen towards W4 is more local emission. It is expected that structures in the local emission will have an angular scale that is larger than structures in the Perseus Arm.

There are two complications to this picture. First, the bright and compact source W3 at $(133.8,+1.2)$ creates imaging artefacts, seen as rings superimposed on W4. Secondly, there is a foreground object superimposed on W5, evident in Fig. 11 as a pink $\left(L_{1.5} \approx\right.$ $0.2 \mathrm{~K})$ ring at $(137.5,+1.1)$. Gray et al. (1998) demonstrate that this lens-like object is either an enhancement of magnetic field or, more likely, an enhancement of electron density, and conclude that it lies somewhere along the $2 \mathrm{kpc}$ line of sight between W5 and the Sun.

Many H II regions other than W4 and W5 in the vicinity show similar behaviour: all of them depolarize distant emission, leaving only foreground polarized emission that has smooth structure (Landecker et al. 2010). This extends as far as $\ell=173^{\circ}$, where the same depolarization effect is seen against the G173+1.5 star formation complex in the Perseus Arm (distance $1.8 \mathrm{kpc}$; Kang, Koo $\&$ Salter 2012).

The region LBN 0679 is a bright filament in total intensity that runs from $\left(141^{\circ},-1.5^{\circ}\right)$ to $\left(140^{\circ}, 0^{\circ}\right)$. It is associated with $\mathrm{H}$ at $-40 \pm 1.5 \mathrm{~km} \mathrm{~s}^{-1}$ (Green 1989). Other H II regions in the vicinity with similar velocities are at a distance around $0.7 \mathrm{kpc}$ (Foster \& Brunt 2015). LBN 0679 shows the same polarization signature: on the $\mathrm{H}$ II region the polarized intensity is smooth, while to either side it is highly structured.

It is evident from Figs $4-7,10$ and 11 that there is strong depolarization in the plane at $1.5 \mathrm{GHz}$. There is an area of depolarization extending over $128^{\circ}<\ell<140^{\circ},-1^{\circ}<b<+2^{\circ}$. In the CGPS data, the southern edge of this depolarization zone has a very sharp edge, barely resolved at arcminute resolution, evident in Fig. 11 as a curved transition region from pink/white to green/black along $b=-1.5$. We refer to this sharp edge as 'the Smile'; it is probably a shock front. Morphological evidence suggests that the Smile is related to W4 and so is on the near side of the Perseus Arm, 
although attempts to associate the Smile with any observed kinematic features have failed (Landecker et al. 2010). In Fig. 10, we trace the polarized intensity at $\ell \approx 135^{\circ}$ as a function of $b$. We see that $L_{1.5} \approx 0.4 \mathrm{~K}$ at $b<-2^{\circ}$. At $b=-1.5$, the polarized intensity drops abruptly to $L_{1.5} \approx 0.2 \mathrm{~K}$. The polarized intensity is similar from $-1.5 \lesssim b \lesssim-0.5$ while the structured appearance we discuss above is evident in Fig. 11. At $b \gtrsim-0.5$, the W4 $\mathrm{H} \alpha$ and $2.7 \mathrm{GHz}$ emission becomes significant and the polarized intensity increases to $L_{1.5} \approx 0.45 \mathrm{~K}$.

We interpret the region in front of W4 and the Smile as follows. W4 completely depolarizes all background emission by beam depolarization, even with the small CGPS beam. The observed $\approx 0.45 \mathrm{~K}$ emission in the direction of W4 is entirely from the foreground and is therefore smooth. The Smile is outside the $\mathrm{H}_{\text {II }}$ region and provides a much lower column of ionized gas than W4. The depolarization in this region is therefore most likely Faraday screen depolarization within the Smile. Because the emission is more distant, it is more structured than the foreground emission observed in front of W4. This interpretation is supported by the fact that there is no depolarization evident in the Smile at $23 \mathrm{GHz}$; if the Smile were geometrical depolarization, we would expect to see depolarization at $23 \mathrm{GHz}$.

Of particular interest is Sh2-202 around $\left(140^{\circ},+2^{\circ}\right)$, diameter 170 arcmin, at a distance of $0.97 \pm 0.08 \mathrm{kpc}$ (Foster \& Brunt 2015). Wilkinson \& Smith (1974) noted that this H II region does not depolarize emission at $610 \mathrm{MHz} .{ }^{4}$ They took this as evidence that the Fan Region emission arises at a distance less than that of Sh2-202. In the CGPS data, we see that Sh2-202 does leave an imprint on the $1.4 \mathrm{GHz}$ polarized intensity image (fig. 7 of Landecker et al. 2010), so at least some of the polarized emission must originate beyond the distance of Sh2-202. Again, the polarized emission across the face of Sh2-202 is smooth and probably local in origin, while that from its surroundings has the more typical mottled appearance that we identify with Perseus Arm emission.

Why can the influence of Sh2-202 as a Faraday screen be detected in the polarization image, when Sh2-202 is not a bright emitter in total intensity? The telescope is more sensitive to the Faraday rotation of a volume of ionized gas than to its bremsstrahlung in the presence of a typical interstellar magnetic field (Uyaniker et al. 2003). Based on the integrated $\mathrm{H} \alpha$ intensity of $37 \mathrm{R}$, we estimate $n_{\mathrm{e}}=1.6 \mathrm{~cm}^{-3}$ in Sh2-202. Assuming a line-of-sight magnetic field of $2 \mu \mathrm{G}$, this yields $\phi \approx 100 \mathrm{rad} \mathrm{m}^{-2}$. This produces a Faraday rotation in the $\mathrm{H}_{\text {II }}$ region at $1420 \mathrm{MHz}$ of $\Delta \psi \approx 250^{\circ}$, easily sufficient to cause Faraday screen depolarization.

These results from the CGPS seem at first sight to be incompatible with the results of Wilkinson \& Smith (1974). However, their conclusions are based on a map of the Fan Region at $610 \mathrm{MHz}$. In Section 4.1, we showed that the appearance of the Fan Region below $1 \mathrm{GHz}$ is quite different from that at $1.5 \mathrm{GHz}$ and above, with the low-frequency emission associated entirely with a nearby feature.

\subsection{Comparison to the W4 superbubble}

Above W4 is the W4 'chimney' or superbubble (Normandeau, Taylor \& Dewdney 1996). The superbubble walls are clearly seen in total intensity at $4.8 \mathrm{GHz}\left(I_{4.8}\right)$. In Fig. 12 , we superimpose $I_{4.8}$ contours on the map of $L_{1.5}$. The $I_{4.8}$ contours fit inside the depression in $L_{1.5}$, strong evidence that the W4 superbubble is responsible for

\footnotetext{
${ }^{4}$ Note that there is a $10^{\circ}$ error in the longitude scale of fig. 5 in Wilkinson \& Smith (1974)
}

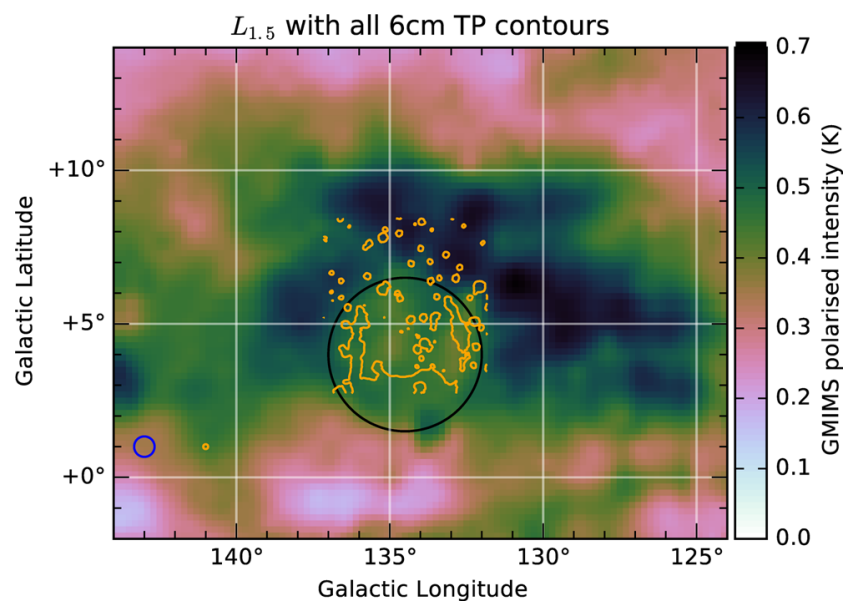

Figure 12. $1.5 \mathrm{GHz}$ polarized continuum from GMIMS [as in Figs 5(b) and 7] with orange contours of $4.8 \mathrm{GHz}$ total power in the $\mathrm{W} 4$ superbubble region from Gao et al. $(2010,2015$ ) (as in Fig. 11). The beams of the $1.5 \mathrm{GHz}$ and $4.8 \mathrm{GHz}$ observations are shown in blue and orange, respectively, in the lower left corner.

the depolarization feature centred at $\left(134.5,+4^{\circ}\right)$. The relative locations of the wall seen at $4.8 \mathrm{GHz}$ and the peak of the $1.5 \mathrm{GHz}$ polarized intensity are somewhat uncertain due to the lower angular resolution of the GMIMS data. The outer edges of the $I_{4.8}$ contours from the superbubble walls are shown as vertical orange lines in Fig. 9. Again, it is clear that the $I_{4.8}$ superbubble walls are inside the region of low $L_{1.5}$. The gradient in $L_{1.5}$ as a function of longitude is much shallower than the gradient in $I_{4.8}$, likely at least partly due to the much lower angular resolution of the $1.5 \mathrm{GHz}$ observations (40 arcmin versus 9.5 arcmin). However, the separation between the peak of $L_{1.4}$ and the edge of the $I_{4.8}$ wall is $\approx 2^{\circ}$ on both sides of the superbubble, comfortably larger than the resolution of the $1.5 \mathrm{GHz}$ observations. Therefore, the placement of the superbubble wall inside, not coincident with, the reduced $L_{1.5}$ emission does not appear to be a resolution effect. The total intensity maps at $1.4 \mathrm{GHz}$ (West et al. 2007; Gao et al. 2015, hereafter GRR15) show that the compressed walls of the superbubble (seen at $4.8 \mathrm{GHz}$ ) lie inside a thicker envelope that is presumably ionized gas. The extent of this ionized material probably determines the extent of the depolarization seen in the GMIMS data. Since W4 is on the near side of the Perseus Arm, this is clear evidence that at least some of the Fan Region emission must be generated in the arm or beyond it.

GRR15 modelled the polarized emission along this line of sight accounting for depth depolarization due to the W4 superbubble. They modelled the superbubble as a shell structure with an inner radius of $65 \mathrm{pc}$ and an outer radius of $72 \mathrm{pc}$. The shell walls are evident as vertical structures at $\ell \approx 136^{\circ}$ and $\ell \approx 133^{\circ}$ in Fig. 12 . The GMIMS beam is $\approx 23 \mathrm{pc}$ at a distance of $2.0 \mathrm{kpc}$, so we do not resolve the shell wall. The West et al. (2007) DRAO Synthesis Telescope data (which lack zero-spacing data and thus are not sensitive to degree-scale structure) show depolarization of $0.025 \mathrm{~K}$ in the shell walls relative to the $\approx 0.5 \mathrm{~K}$ large-scale polarized brightness temperature at $\lambda=21 \mathrm{~cm}$. In Fig. 12, the shell walls are inside the edge of the 0.7 K Fan Region emission we see with GMIMS. GRR15 concluded that the depolarization by the shell walls is only $\approx 5$ per cent at $21 \mathrm{~cm}$.

Because the $\gtrsim 1^{\circ}$-scale structure in the $1.4 \mathrm{GHz}$ polarization images presented by GRR15 is tied to the Wolleben et al. (2006) survey (which does not show the large depolarization feature in the Fan Region, as we discussed in Section 4.1), the lack of the large 
depolarization feature in the data presented by GRR15 is expected. Our result is therefore not inconsistent with GRR15. The drop in intensity from the Fan Region outside the shell wall $\left(L_{1.5} \approx 0.7 \mathrm{~K}\right)$ to inside the shell $\left(L_{1.5} \approx 0.5 \mathrm{~K}\right)$ is evident with the GMIMS observations and indicates that the superbubble as a whole depolarizes the Fan Region emission by $\approx 30$ per cent; this is the depolarization that is not evident in the GRR15 data. Then the superbubble wall depolarizes the $0.5 \mathrm{~K} 1.4 \mathrm{GHz}$ emission on $\sim 10$ arcmin scales by $\approx 5$ per cent; the GMIMS data are not sensitive to variations in intensity on angular scales this small.

\section{DISCUSSION}

\subsection{Polarization fraction}

The Fan Region has exceptionally high polarized intensity, but the Stokes $I$ emission is typical of the surrounding parts of the Galaxy, $I_{1.5} \approx 1.6 \mathrm{~K}$. Equivalently, the polarization fraction in the Fan Region is unusually high, $\approx 40$ per cent (Fig. 6). Aside from the North Polar Spur, no other region of the sky has such strong emission with such high fractional polarization. We have argued that $\gtrsim 30$ per cent of the $1.5 \mathrm{GHz}$ polarized emission originates in or beyond the Perseus Arm through a morphological comparison of $L_{1.5}$ to $\mathrm{H} \alpha$ and $I_{4.8}$ observations of ionized gas in the Perseus Arm (Sections 4.2-4.4). However, we can argue for the same conclusion from the polarized radio continuum data alone. Bingham \& Shakeshaft (1967) were the first to do this, and their argument, using modern data, is as follows.

The maximum possible polarized fraction of synchrotron emission is 70 per cent (Ginzburg \& Syrovatskii 1965), so in the simplest model, $\sim 4 / 7$ of the radio continuum-emitting portion of the sightline contributes to the Fan Region signal. (That estimate must, of course, take into account the varying synchrotron emissivity along lines of sight through the Galaxy, as we do in Section 5.3.) Cosmic rays and magnetic fields, the ingredients which produce synchrotron emission, are well distributed around the Galaxy, so the Stokes I emission is produced along a long (at least several $\mathrm{kpc}$ ) path. If $4 / 7$ of the path contributes to the polarized emission, the polarized emission must also be produced along a long path.

GRR15 applied the Sun et al. (2008) model of Galactic synchrotron emission to the W4 (and Fan Region) sightline. This model incorporates an enhanced synchrotron emissivity near the Sun (Fleishman \& Tokarev 1995). Fig. 5 of GRR15 shows that, in this model, $\approx 50$ per cent of the Stokes $I$ emission originates within $1 \mathrm{kpc}$ of the Sun and $\approx 25$ percent originates within $500 \mathrm{pc}$. This figure describes the $4.8 \mathrm{GHz}$ emission, but assuming that the spectral index is constant - which we expect for Stokes $I$, which does not suffer from Faraday depolarization - the same fractions should apply for $I_{1.5}$. If all of the Fan Region polarized emission that reaches our telescope originates within $500 \mathrm{pc}(1 \mathrm{kpc})$ and the emitted fractional polarization is the maximal 70 per cent, the expected polarization fraction is therefore 18 percent ( 35 per cent). Therefore, the observed fractional polarization cannot be explained by emission within $500 \mathrm{pc}$ of the Sun, and the fractional polarization in this model within $1 \mathrm{kpc}$ of the Sun is still somewhat lower than (although probably within the uncertainties of) the observed fractional polarization.

\subsection{Source of the synchrotron emission}

What is the source of the emission? Synchrotron emission requires cosmic rays and a component of the magnetic field perpendicular to the line of sight. The scaleheights of both cosmic rays and the regular component of the magnetic field are highly uncertain but of the order of a few kpc (Ferrière 2001), so there is no difficulty in finding the ingredients required to generate synchrotron emission at moderate latitudes. In addition to the morphological depolarization argument we presented in Section 4, there are then two lines of argument that suggest that a significant component of the emission is within the Perseus Arm or in the interarm region beyond the arm.

First, the Fan Region as seen at 1.5, 23 and $353 \mathrm{GHz}$ extends from $b \approx-5^{\circ}$ to $b \approx+10^{\circ}$ (Section 4.2 and Figs 4,5 and 10 ), centred above the plane in a part of the Galaxy in which the emission beyond the Perseus Arm is warped upward to $b \approx+5^{\circ}$ (Section 3 and Fig. 3). If the warp explains the asymmetry of the Fan Region about the plane, some of the emission must be beyond the Perseus Arm.

Secondly, the magnetic field within the volume that generates the Fan emission must be uniform to produce the highly ordered polarization signal. In an interarm region, the magnetic field is more likely to remain coherent over the long path length required to produce uniform synchrotron emission, and this may argue for at least a partial origin of the Fan Region emission in regions beyond the Perseus Arm. There is evidence for a more ordered field in interarm regions in the face-on spirals M51 and NGC 6946 (Beck 2007; Fletcher et al. 2011).

We therefore conclude that the most likely explanation for the Fan Region emission is geometric. The Fan Region is the portion of the Galaxy in which there is both a long path length with a coherent magnetic field, with a significant component perpendicular to the line of sight, and a warp that allows us to see much of the path length around depolarizing variations in the foreground gas. In Section 5.3, we construct a simple model applying this qualitative discussion.

\subsection{Spiral structure and synchrotron emission}

A number of authors have constructed models of the Galactic magnetic field aiming to fit a number of observational constraints, including diffuse polarized emission. Some mask the Fan Region from their fits (e.g. Jansson \& Farrar 2012), while others ignore it, leaving high residuals (e.g. Sun et al. 2008; Jaffe et al. 2010, 2011). In general, these models include a regular, planar field with a logarithmic spiral with a pitch angle of the same sign as the spiral defining the gaseous and stellar spiral structure ${ }^{5}$ and a value of $8^{\circ}$ $\lesssim \Theta \lesssim 12^{\circ}$. Van Eck et al. (2011) argue for an azimuthal field $(\Theta$ $\approx 0^{\circ}$ ) in the outer Galaxy. The models also typically include turbulent, random or striated fields; a vertical field and contributions from discrete structures. Although these models have had varying degrees of success in matching the observed features in the Fan Region at low frequencies $(v \lesssim 2 \mathrm{GHz}$ ), none have fit it with low residuals as a global feature. However, a spiral magnetic field with a positive pitch angle places the maximal polarized intensity in the second quadrant $\left(\ell<180^{\circ}\right)$ because the perpendicular component of the magnetic field is larger there than at $\ell=180^{\circ}$ or in the third quadrant. Models developed in preparation for the Planck mission account for the $353 \mathrm{GHz}$ polarized emission from the Fan Region with only a global spiral magnetic field and turbulence feature a maximum in polarization fraction around $\ell=155^{\circ}$ (fig. 7 of Miville-Deschênes et al. 2008), in the second quadrant though at a higher longitude than the Fan Region. These models too have high

\footnotetext{
${ }^{5}$ Jansson \& Farrar (2012) define this pitch angle sign as positive, while Sun et al. (2008) define this sign as negative; we choose positive.
} 
residuals in polarization in the Fan Region (Delabrouille et al. 2013; Planck Collaboration XIX 2015; Planck Collaboration XLII 2016).

We have attempted a very simple model that suggests one possible explanation while illustrating the problems that still need to be solved before an adequate, complete model of the Fan Region emission can be devised. Our model (inspired by Jansson \& Farrar 2012 and Sun et al. 2008) includes only a radial variation in the magnetic field, excluding reversals (which are irrelevant to the calculation of synchrotron emissivity, which is proportional to $|\boldsymbol{B}|^{2}$ ), the vertical component of the field, and separate spiral arms. We assume that the magnetic field is

$\boldsymbol{B}=\frac{\boldsymbol{B}_{0}}{r_{\mathrm{GC}}} \hat{b}$

where $r_{\mathrm{GC}}$ is the distance from the Galactic Centre to the position, the unit vector along the logarithmic spiral defining the magnetic field is $\hat{b}=\sin (\Theta) \hat{r}+\cos (\Theta) \hat{\phi}$ in Galactocentric cylindrical coordinates, and the magnetic logarithmic spiral has a pitch angle $\Theta$. We further assume that the cosmic ray electron density is (following Page et al. 2007)

$n_{\text {cre }}=n_{\text {cre }, 0} \mathrm{e}^{-r_{\mathrm{GC}} / h_{r}} \operatorname{sech}^{2}\left(z / h_{z}\right)$,

where $h_{r}=5 \mathrm{kpc}$ and $h_{z}=1 \mathrm{kpc}$ are the scalelength and height of the cosmic ray electron distribution. At $r_{\mathrm{GC}}>20 \mathrm{kpc}$, we set $B$ and $n_{\text {cre }}$ to zero. We model the thermal electron density outside the Solar circle as

$$
\begin{aligned}
n_{\mathrm{e}}= & n_{1} \frac{\operatorname{sech}^{2}\left(r_{\mathrm{GC}} / A_{1}\right)}{\operatorname{sech}^{2}\left(r_{\odot} / A_{1}\right)} \exp \left(-\frac{|z|}{h_{1}}\right) \\
& +n_{a} \operatorname{sech}^{2}\left(\frac{z}{h_{a}}\right) e^{-\left(s_{\mathrm{Per}} / w_{a}\right)^{2}} \operatorname{sech}^{2}\left(\frac{r_{\mathrm{GC}}-r_{\odot}}{2.0 \mathrm{kpc}}\right) .
\end{aligned}
$$

The first term is the smooth component of the Taylor \& Cordes (1993) model of the Galactic electron density modified to use an exponential vertical distribution (Schnitzeler 2012). We choose $h_{1}=1.4 \mathrm{kpc}$ and $h_{1} n_{1}=0.022 \mathrm{kpc} \mathrm{cm}^{-3}$, appropriate for the solar neighbourhood (Gaensler et al. 2008; Savage \& Wakker 2009), and $A_{1}=20 \mathrm{kpc}$. The second term represents the contribution from the Perseus spiral arm. We use the Reid et al. (2014) definition of the arm as a logarithmic spiral (Fig. 1); $s_{\mathrm{Per}}$ is the distance from a given position to the nearest point in the spiral arm, $n_{a}=0.084 \mathrm{~cm}^{-3}$ is the mid-plane electron density in the arm and $w_{a}=300 \mathrm{pc}$ is the assumed scalelength of the electron density in the arm (Taylor \& Cordes 1993).

For each longitude, we calculate the polarized synchrotron intensity from the polarization vector (Burn 1966; Sokoloff et al. 1998; O'Sullivan et al. 2012)

$\mathcal{P}=\int_{\text {back }}^{\text {observer }} \varepsilon n_{\text {cre }}(s)(\boldsymbol{B}(s) \times \hat{s})^{2} e^{2 i \psi(s, \lambda)} \mathrm{d} s$

where the polarization angle is $\psi(s, \lambda)=\psi_{0}(s)+\phi(s) \lambda^{2}$ and the Faraday depth is defined in equation (1). We use equations (2)-(4) to determine $n_{\text {cre }}, n_{\mathrm{e}}$ and $\boldsymbol{B}$. We choose the emissivity $\varepsilon$ such that $n_{\text {cre, } 0} \varepsilon=0.7 \mathrm{~K} \mathrm{~cm}^{-3}$ because this produces a synchrotron intensity in the Fan Region of $\approx 0.5 \mathrm{~K}$, as observed, but the units of the output intensity can be scaled arbitrarily. We stay in the Galactic plane $(z=0 \mathrm{kpc})$. This implicitly but inexactly accounts for the warp: because the brightest $1.5 \mathrm{GHz}$ emission in the Fan Region has a non-zero latitude $\left(b \approx+8^{\circ}\right)$, the altitude probed by the sightline would increase with distance as $d \sin b$. In a warped disc, the latitude of the mid-plane increases with distance, so the distance between the sightline and the mid-plane is less than $d \sin |b|$. The

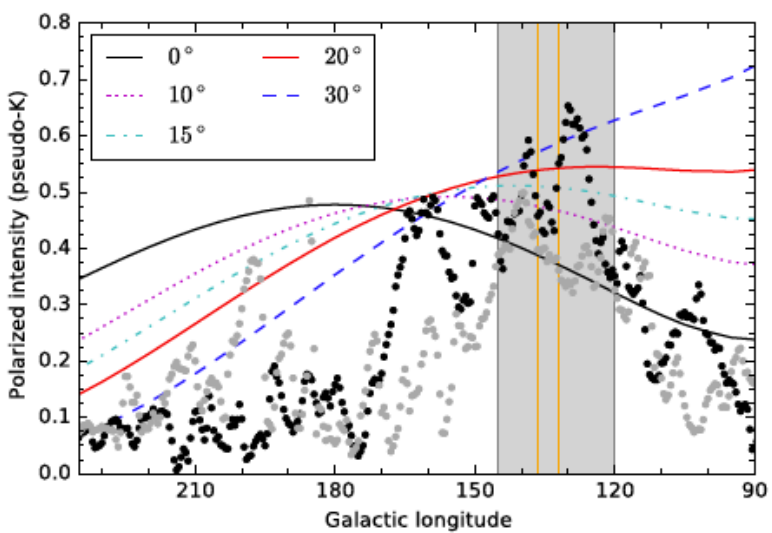

Figure 13. Synthetic polarized synchrotron intensity at $\lambda=20 \mathrm{~cm}$ as a function of longitude for a range of magnetic spiral pitch angles $\Theta$ as described in Section 5.3. This model includes depth and geometrical but not beam depolarization. As in Fig. 1, the longitude range of the Fan Region is shaded grey. We also show polarized intensity from GMIMS-HBN as a function of Galactic longitude at $b=+4.5$ (black dots) and $b=-4.5$ (light grey dots). The orange lines denote the outer edge of the W4 superbubble as in Fig. 9.

inclusion of equations (1) and (4) accounts for depth depolarization and equation (5) includes geometrical depolarization, but we leave the inclusion of beam depolarization for Paper II because it involves a number of additional assumptions that are more difficult to quantify.

We show this modelled mid-plane polarized synchrotron intensity as a function of Galactic longitude in the outer Galaxy in Fig. 13. The intensity is maximal at $\ell=180^{\circ}$ for $\Theta=0^{\circ}$ (a circular field). For non-zero pitch angles, the polarized synchrotron intensity is much higher in the second quadrant $\left(\ell<180^{\circ}\right)$ than in the third quadrant $\left(\ell>180^{\circ}\right)$ due to the small perpendicular component of $\boldsymbol{B}$ in the third quadrant. In the second quadrant, absent depth depolarization effects, the polarized synchrotron intensity is relatively flat as a function of longitude when $\Theta>0$. However, due to depth depolarization, the observed polarized synchrotron intensity reaches a maximum and then decreases towards lower longitude. The longitude of the peak is lower for higher $\Theta$; for $15^{\circ} \lesssim \Theta \lesssim 20^{\circ}$, the intensity peaks in the Fan Region. For $\Theta=30^{\circ}$, the intensity does not peak in the second quadrant; it increases monotonically from $\ell=180^{\circ}$ to $\ell=90^{\circ}$.

We also show polarized intensity as a function of longitude in the entire portion of the outer Galaxy observed with GMIMS-HBN in Fig. 13. We show $L_{1.5}$ at $b=+4.5$, which runs through the centre of the Fan Region, and $b=-4.5$, which runs through the brightest polarized emission in the third quadrant (see Fig. 4a). The longitude of peak emission from the $\Theta \approx 20^{\circ}$ models roughly matches that in the observations. However, the observed polarized intensity falls off much more rapidly on either side of the peak than these models but more slowly than the $\Theta=0^{\circ}$ model. In particular, the modelled $\Theta=20^{\circ}$ polarized intensity is close to flat at $\ell<130^{\circ}$.

The addition of a source of cosmic ray electrons concentrated around W4 can produce a peak in polarized intensity that roughly matches the observed cross-section through the Fan Region shown in Fig. 13, but (a) there is no observational evidence, in the way of supernova remnants, for any source of high-energy electrons and (b) this extra synchrotron emissivity would produce a peak in total intensity as well, which is not observed. The puzzling feature of the Fan Region is its exceptionally high polarized intensity, not its total intensity. The remaining difficulty is to explain the very high level 
of uniformity of the magnetic field implied by the high fractional polarization, and to explain that field regularity over the long path length that is implied by the observational evidence presented in this paper.

None of these simple models accurately match the morphology of the Fan Region. Models with a pitch angle $\Theta \approx 10^{\circ}$, as others have generally preferred (e.g. Miville-Deschênes et al. 2008; Sun et al. 2008; Jansson \& Farrar 2012) produce a peak in intensity at higher longitudes than is observed in the Fan Region, while the steep pitch angle which best matches the longitude of peak intensity produces a higher intensity than is observed to lower longitudes than the Fan Region.

We conclude that it is unlikely that any simple model of the Galactic magnetic field will explain all of the observations. However, a spiral magnetic field with a relatively steep pitch angle in the outer Galaxy can plausibly explain a distant origin of $\approx 30$ per cent of the Fan Region emission. The Fan Region is in the quadrant of the Galaxy one would expect for a feature that arises due to synchrotron emission from the Galactic magnetic field with a spiral with a positive pitch angle. We note that the gas in the Outer Arm has a steep pitch angle, $\Theta=18.6$ (Fig. 1 and Reid et al. 2014). It is possible that the Fan Region originates in a part of the Galaxy with a steeper pitch than is preferred at $r_{\mathrm{GC}} \lesssim 10 \mathrm{kpc}$, although this result is inconsistent with the azimuthal field towards the anticentre preferred by Van Eck et al. (2011) based on RM measurements of extragalactic sources.

\section{SUMMARY AND CONCLUSIONS}

We have used new GMIMS-HBN observations and other published observations to describe the morphology of polarized continuum emission found in data over $0.4-353 \mathrm{GHz}$ in the Fan Region. In summary, our key observational findings are as follows.

(i) All-sky maps of 1.5, 23 and $353 \mathrm{GHz}$ polarized emission from the Fan Region show that the Fan Region is roughly coincident with $\mathrm{H} \alpha$ emission from the Perseus Arm, especially the $\mathrm{H} \alpha$ emission from around the W3/W4/W5 complex of $\mathrm{H}$ II regions. They are similar in both location and angular extent on $\approx 10^{\circ}$ scales [Figs $4,5(\mathrm{a})$ and (b)].

(ii) While the large-scale extent of the Fan Region polarized emission at $>1 \mathrm{GHz}$ roughly coincides with the Perseus Arm $\mathrm{H} \alpha$ emission, the detailed structure on $\approx 1^{\circ}$ scales shows anticorrelation. A morphological comparison of GMIMS-HBN data at $1.5 \mathrm{GHz}$ to $\mathrm{H} \alpha$ (Figs 7-9 and Section 4.2) and radio continuum total intensity observations with high angular resolution (Figs 11 and 12 and Section 4.3) shows that depolarization evident in polarized intensity is correlated with bright features in ionized gas related to the W4 star formation region and superbubble. The polarized intensity $L_{1.5}$ is 30 per cent lower in regions with high integrated $\mathrm{H} \alpha$ intensity from the Perseus Arm $\left(I_{\mathrm{H} \alpha}>5 \mathrm{R}\right)$ than in regions with low integrated $\mathrm{H} \alpha$ intensity from the Perseus Arm $\left(I_{\mathrm{H} \alpha}<3\right.$ R; Fig. 8).

(iii) At frequencies lower than $1.5 \mathrm{GHz}$, the size of the Fan Region decreases with decreasing frequency. At $v \lesssim 600 \mathrm{MHz}$, the morphology of the Fan Region is quite different than at $v \gtrsim 1 \mathrm{GHz}$ : the low-frequency emission is a ring centred at $\left(137^{\circ},+8^{\circ}\right)$, while the high-frequency emission extends to significantly lower longitudes $\left(\ell \approx 115^{\circ}\right)$ and latitudes $\left(b \approx-5^{\circ}\right)$ and does not have a ring-like component (Section 4.1 and Fig. 5).

(iv) The fractional polarization of the parts of the Fan Region with bright polarized emission at $1.5 \mathrm{GHz}$ is high, $L_{1.5} / I_{1.5} \approx 40$ per cent (Section 4.1 and Fig. 6).
Observational fact (ii) leads us to conclude that at least 30 per cent of the $1.5 \mathrm{GHz}$ polarized continuum emission seen in the brightest parts of the Fan Region originates in or beyond the Perseus Arm. If the Perseus Arm acts as a Burn (1966) slab that depolarizes all background emission - a conclusion supported by the smoothness of the CGPS data - most or all of the remaining $\approx 70$ per cent would originate in front of the Perseus Arm. Observational fact (iii) suggests that this high-frequency structure is different in physical origin than most of the low-frequency ( $v \lesssim 600 \mathrm{MHz}$ ) emission that was first associated in the literature with the Fan Region. The more distant high-frequency emission is likely more depolarized at low frequencies (which we will discuss more in Paper II), so the emission and Faraday effects in local features, within about $500 \mathrm{pc}$, may dominate. These local features appear to make an insignificant contribution to the Fan Region at high frequencies. The rest of our conclusions apply to the $v>1 \mathrm{GHz}$ data.

Observational fact (iv) implies that the entire line of sight must be involved in generating the Fan Region synchrotron emission (Section 5.2). Even though a majority (up to 70 percent) of the $1.5 \mathrm{GHz}$ emission could originate in front of the Perseus Arm, it seems highly unlikely that most of the foreground emission is within $\approx 500$ pc while 30 percent of the emission originates in or beyond the Perseus Arm. It is far more plausible that the origin of the Fan Region is not a discrete, local structure. Therefore, the Fan Region must be a very large phenomenon, several kpc in extent, which we can only explain as a consequence of Galactic structure and geometry. We cannot confine it, as most previous authors have done, to the nearest $500 \mathrm{pc}$. We could have reached many of our conclusions about the distance of the Fan Region without the GMIMS data or the $\mathrm{H} \alpha$ data based entirely on the high fractional polarization, the modelling of Section 5.3 and the evidence from the Planck $353 \mathrm{GHz}$ data, together with the modelling done by the Planck consortium (see references in Section 5.3). The GMIMS and WHAM data, taken together, reinforce our conclusion.

Our determination of the distance to the Fan Region emission from the GMIMS and WHAM data is strongly supported by the correlation of the $\approx 4^{\circ}$-diameter region of reduced polarized intensity around $\left(134.5,+4^{\circ}\right)$ with $\mathrm{H} \alpha$ intensity at Perseus Arm velocities and the W4 superbubble. This is a relatively small patch of sky. Our extrapolation from the Fan Region to an analysis of the Galactic magnetic field rests on analysis of observations over much larger scales, the fact that the polarized intensity is much higher in the second quadrant (where the Fan Region extends over $\sim 60^{\circ}$ ) than in the third quadrant.

We suggest following three ideas that explain some of the observed features of the Fan Region emission

(i) A spiral magnetic field with a steep pitch angle $\left(\Theta \approx 15^{\circ}\right.$ to $20^{\circ}$ ) moves the longitude of peak emission to $\ell \approx 130^{\circ}$ in the Fan Region (Fig. 13). However, the increase in intensity is less sharp than observed, and the pitch angle is significantly steeper than the $\Theta \approx 10^{\circ}$ preferred by most existing models.

(ii) An increase in the synchrotron emissivity associated with W4 could be scaled to produce the observed Fan Region intensity. Qualitatively, this is consistent with the morphology of the brightest emission from the Fan Region, surrounding W4. However, the $60^{\circ} \times 30^{\circ}$ extent of the Fan Region is much larger than W4 itself. Moreover, it is not obvious how to increase the polarized intensity without also increasing the total intensity, and it is also not obvious how increased intensity in a presumably turbulent region associated with star formation could produce such regular polarization vectors 
and such a high polarization fraction. This idea also does not explain the offset of the Fan Region above $b=0^{\circ}$.

(iii) Due to the warp, distant $(d>2 \mathrm{kpc})$ portions of this part of the Galaxy are centred at $b=+3^{\circ}$ to $+9^{\circ}$ (Fig. 3). Moreover, the pitch of the gaseous arms is considerably steeper in the outer Galaxy (Fig. 1); because most models of the magnetic field are not constrained so far out $(R \gtrsim 20 \mathrm{kpc})$, a steeper pitch angle may be consistent with existing models. It is not clear that the cosmic ray electron density is high enough in the outer Galaxy, where there is little star formation, to produce the observed synchrotron intensity. However, in M51, there is detectable polarized emission at $1.4 \mathrm{GHz}$ out to $\sim 50 \mathrm{kpc}$ from the galactic centre, well beyond the optical spiral arms (Fletcher et al. 2011).

None of these three ideas explain all of the observed features of the Fan Region, so the origin of the Fan Region remains puzzling. We conclude that $\approx 30$ per cent of the integrated $1.5 \mathrm{GHz}$ Fan Region emission is depolarized by ionized gas in the Perseus Arm, suggesting that it is a puzzling Galactic-scale feature, not a relatively small, purely local feature. This result suggests that future detailed models of the Galactic magnetic field should attempt to fit the Fan Region with the prior that the emission originates along a long path length or at a large distance, perhaps incorporating the warp or a spiral magnetic field with a large pitch angle in at least part of the Galaxy.

\section{ACKNOWLEDGEMENTS}

ASH acknowledges useful discussions with R. A. Benjamin and V. Jelic. TLL acknowledges useful discussions with T. Foster and J. C. Brown. We thank two referees for comments that strengthened the paper.

This research made use of APLpy ${ }^{6}$ and Astropy (Astropy Collaboration et al. 2013).

The Wisconsin H-Alpha Mapper is funded by the US National Science Foundation. The DRAO is operated as a National Facility by the National Research Council Canada. ASH was partially supported by NSF grant AST-1442650 and grant HST-AR-14297, provided by NASA through a grant from the Space Telescope Science Institute, which is operated by the Association of Universities for Research in Astronomy, Incorporated, under NASA contract NAS5-26555. KD and MW were supported by the Natural Sciences and Engineering Research Council of Canada. BMG acknowledges the support of the Australian Research Council through grant FL100100114. The Dunlap Institute is funded through an endowment established by the David Dunlap family and the University of Toronto. NMM-G acknowledges the support of the Australian Research Council through Future Fellowship FT150100024. MH acknowledges the support of research program 639.042.915, which is partly financed by the Netherlands Organization for Scientific Research (NWO).

\section{REFERENCES}

Astropy Collaboration et al., 2013, A\&A, 558, 33

Baars J. W. M., Genzel R., Pauliny-Toth I. I. K., Witzel A., 1977, A\&A, 61, 99

Beck R., 2007, A\&A, 470, 539

\footnotetext{
${ }^{6}$ http://aplpy.github.com
}

Benjamin R. A., 2008, in Henrik Beuther, Hendrik Linz, and Thomas Henning, eds, ASP Conf. Ser., Vol. 387, Massive Star Formation: Observations Confront Theory. Astron. Soc. Pac., San Francisco, p. 375

Bennett C. L. et al., 2013, ApJS, 208, 20

Berkhuijsen E. M., Brouw W. N., Muller C. A., Tinbergen J., 1964, Bull. Astron. Inst. Neth., 17, 465

Bernardi G. et al., 2009, A\&A, 500, 965

Bingham R. G., Shakeshaft J. R., 1967, MNRAS, 136, 347

Brentjens M. A., de Bruyn A. G., 2005, A\&A, 441, 1217

Brouw W. N., Spoelstra T. A. T., 1976, A\&AS, 26, 129

Burn B. J., 1966, MNRAS, 133, 67

Calabretta M. R., Greisen E. W., 2002, A\&A, 395, 1077

Carretti E., Bernardi G., Sault R. J., Cortiglioni S., Poppi S., 2005, MNRAS, 358, 1

Churchwell E. et al., 2009, PASP, 121, 213

Delabrouille J. et al., 2013, A\&A, 553, 96

Du X., Landecker T. L., Robishaw T., Gray A. D., Douglas K. A., Wolleben M., 2016, PASP, 128, 115006

Ferrière K. M., 2001, Rev. Mod. Phys., 73, 1031

Fleishman G. D., Tokarev Y. V., 1995, A\&A, 293, 565

Fletcher A., Beck R., Shukurov A., Berkhuijsen E. M., Horellou C., 2011, MNRAS, 412, 2396

Foster T., Brunt C. M., 2015, AJ, 150, 147

Foster T., MacWilliams J., 2006, ApJ, 644, 214

Gaensler B. M., Dickey J. M., McClure-Griffiths N. M., Green A. J., Wieringa M. H., Haynes R. F., 2001, ApJ, 549, 959

Gaensler B. M., Madsen G. J., Chatterjee S., Mao S. A., 2008, PASA, 25, 184

Gao X. Y. et al., 2010, A\&A, 515, 64

Gao X. Y., Reich W., Reich P., Han J. L., Kothes R., 2015, A\&A, 578, A24 (GRR15)

Ginzburg V. L., Syrovatskii S. I., 1965, ARA\&A, 3, 297

Gray A. D., Landecker T. L., Dewdney P. E., Taylor A. R., 1998, Nature, 393,660

Gray A. D., Landecker T. L., Dewdney P. E., Taylor A. R., Willis A. G., Normandeau M., 1999, ApJ, 514, 221

Green D. A., 1989, AJ, 98, 2210

Hachisuka K., Brunthaler A., Menten K. M., Reid M. J., Hagiwara Y., Mochizuki N., 2009, ApJ, 696, 1981

Haffner L. M., Reynolds R. J., Tufte S. L., 1999, ApJ, 523, 223

Haffner L. M., Reynolds R. J., Tufte S. L., Madsen G. J., Jaehnig K. P., Percival J. W., 2003, ApJS, 149, 405

Haffner L. M. et al., 2010, ASPC, 438, 388

Haverkorn M., Katgert P., de Bruyn A. G., 2003, A\&A, 404, 233

Heald G. H., 2009, in Strassmeier K. G., Kosovichev A. G., Beckman J. E., eds, Proc. IAU Symp. 259, Cosmic Magnetic Fields: From Planets. Cambridge Univ. Press, Cambridge, p. 591

Iacobelli M., Haverkorn M., Katgert P., 2013a, A\&A, 549, 56

Iacobelli M. et al., 2013b, A\&A, 558, 72

Jaffe T. R., Leahy J. P., Banday A. J., Leach S. M., Lowe S. R., Wilkinson A., 2010, MNRAS, 401, 1013

Jaffe T. R., Banday A. J., Leahy J. P., Leach S., Strong A. W., 2011, MNRAS, 416, 1152

Jansson R., Farrar G. R., 2012, ApJ, 757, 14

Kalberla P. M. W., Burton W. B., Hartmann D., Arnal E. M., Bajaja E., Morras R., Pöppel W. G. L., 2005, A\&A, 440, 775

Kalberla P. M. W., Dedes L., Kerp J., Haud U., 2007, A\&A, 469, 511

Kang J.-h., Koo B.-C., Salter C., 2012, AJ, 143, 75

Kennicutt R. C., Evans N. J., 2012, ARA\&A, 50, 531

Landecker T. L. et al., 2010, A\&A, 520, 80

Madsen G. J., Reynolds R. J., 2005, ApJ, 630, 925

Madsen G. J., Reynolds R. J., Haffner L. M., 2006, ApJ, 652, 401

Miville-Deschênes M. A., Ysard N., Lavabre A., Ponthieu N., Macías-Pérez J. F., Aumont J., Bernard J. P., 2008, A\&A, 490, 1093

Normandeau M., Taylor A. R., Dewdney P. E., 1996, Nature, 380, 687

O'Sullivan S. P. et al., 2012, MNRAS, 421, 3300

Page L. et al., 2007, ApJS, 170, 335

Planck Collaboration I, 2016, A\&A, 594, A1 
Planck Collaboration XIX, 2015, A\&A, 576, A104

Planck Collaboration XLII, 2016, A\&A, 596, A103

Reich W., 1982, A\&AS, 48, 219

Reich P., Reich W., 1986, A\&AS, 63, 205

Reich P., Testori J. C., Reich W., 2001, A\&A, 376, 861

Reid M. J. et al., 2009, ApJ, 700, 137

Reid M. J. et al., 2014, ApJ, 783, 130

Savage B. D., Wakker B. P., 2009, ApJ, 702, 1472

Schnitzeler D. H. F. M., 2012, MNRAS, 427, 664

Schnitzeler D. H. F. M., Katgert P., de Bruyn A. G., 2009, A\&A, 494, 611

Sokoloff D. D., Bykov A. A., Shukurov A., Berkhuijsen E. M., Beck R., Poezd A. D., 1998, MNRAS, 299, 189

Spoelstra T. A. T., 1984, A\&A, 135, 238

Sun X. H., Han J. L., Reich W., Reich P., Shi W. B., Wielebinski R., Fürst E. J., 2007, A\&A, 463, 993

Sun X. H., Reich W., Waelkens A., Enßlin T. A., 2008, A\&A, 477, 573

Sun X. H. et al., 2015, ApJ, 811, 40

Tauber J. A. et al., 2010, A\&A, 520, A1

Taylor J. H., Cordes J. M., 1993, ApJ, 411, 674

Taylor A. R. et al., 2003, AJ, 125, 3145

Tribble P. C., 1991, MNRAS, 250, 726

Uyaniker B., Landecker T. L., Gray A. D., Kothes R., 2003, ApJ, 585, 785
Van Eck C. L. et al., 2011, ApJ, 728, 97

Verschuur G. L., 1968, The Observatory, 88, 15

West J. L., English J., Normandeau M., Landecker T. L., 2007, ApJ, 656, 914

Westerhout G., Seeger C. L., Brouw W. N., Tinbergen J., 1962, Bull. Astron. Inst. Neth., 16, 187

Wielebinski R., Shakeshaft J. R., Pauliny-Toth I. I. K., 1962, The Observatory, 82,158

Wilkinson A., Smith F. G., 1974, MNRAS, 167, 593

Wolleben M., 2005, PhD thesis, Univ. Bonn

Wolleben M., Landecker T. L., Reich W., Wielebinski R., 2006, A\&A, 448, 411

Wolleben M. et al., 2009, in Strassmeier K. G., Kosovichev A. G., Beckman J. E., eds, Cosmic Magnetic Fields: From Planets. Cambridge Univ. Press, p. 89

Wolleben M., Landecker T. L., Hovey G. J., Messing R., Davison O. S., House N. L., Somaratne K. H. M. S., Tashev I., 2010a, AJ, 139, 1681

Wolleben M. et al., 2010b, ApJ, 724, L48

Xu Y., Reid M. J., Zheng X. W., Menten K. M., 2006, Science, 311, 54

This paper has been typeset from a $\mathrm{T}_{\mathrm{E}} \mathrm{X} / \mathrm{L} \mathrm{T}_{\mathrm{E}} \mathrm{X}$ file prepared by the author. 